PAPER

\section{A novel algorithm for the calculation of physical and biological irradiation quantities in scanned ion beam therapy: the beamlet superposition approach}

To cite this article: G Russo et al 2016 Phys. Med. Biol. 61183

View the article online for updates and enhancements.
Related content

- Nuclear physics in particle therapy: a
$\frac{\text { review }}{\text { Marco Durante and Harald Paganetti }}$
- The FLUKA Monte Carlo code coupled
$\frac{\text { with the NIRS approach for clinical dose }}{\text { calculations in carbon ion therapy }}$
G Magro, T J Dahle, S Molinelli et al.
- $\frac{\text { RBE and related modeling in carbon-ion }}{\text { therapy }}$ Christian P Karger and Peter Peschke

Recent citations

- FRoG-A New Calculation Engine for
$\frac{\text { Clinical Investigations with Proton and }}{\text { Carbon lon Beams at CNAO }}$
KyungDon Choi et al
- 'Survival': a simulation toolkit introducing a
$\frac{\text { modular approach for radiobiological }}{\text { evaluations in ion beam therapy }}$
L Manganaro et al
- Tumour control in ion beam radiotherapy
$\frac{\text { with different ions in the presence of }}{\text { hypoxia: an oxygen enhancement ratio }}$
$\frac{\text { model based on the microdosimetric }}{\text { kinetic model }}$
L Strigari et al

\title{
Uncompromised.
}

See clearly during treatment to attack the tumor and protect the patient.

\section{Two worlds, one future.}

\section{CEElekta}




\title{
A novel algorithm for the calculation of physical and biological irradiation quantities in scanned ion beam therapy: the beamlet superposition approach
}

\author{
G Russo ${ }^{1}$, A Attili ${ }^{1}$, G Battistoni ${ }^{2}$, D Bertrand ${ }^{5}$, F Bourhaleb ${ }^{6}$, \\ F Cappucci ${ }^{2}$, M Ciocca ${ }^{7}$, A Mairani ${ }^{7}$, F M Milian ${ }^{1,8}$, \\ S Molinelli ${ }^{7}$, M C Morone ${ }^{3}$, S Muraro ${ }^{2}$, T Orts $^{5}$, V Patera ${ }^{4}$, \\ P Sala $^{2}$, E Schmitt ${ }^{1}$, G Vivaldo ${ }^{1}$ and F Marchetto ${ }^{1}$ \\ ${ }^{1}$ Istituto Nazionale di Fisica Nucleare (INFN), Torino, Italy \\ 2 Istituto Nazionale di Fisica Nucleare (INFN), Milano, Italy \\ ${ }^{3}$ Istituto Nazionale di Fisica Nucleare (INFN) and Università 'Tor Vergata', Roma, \\ Italy \\ ${ }^{4}$ Istituto Nazionale di Fisica Nucleare (INFN) Laboratori Nazionali di Frascati and \\ Università 'La Sapienza', Roma, Italy \\ ${ }^{5}$ Ion Beam Applications (IBA), Louvain-la-Neuve, Belgium \\ ${ }^{6}$ Internet-Simulation Evaluation Envision (I-SEE), Torino, Italy \\ 7 Centro Nazionale di Adroterapia Oncologica (CNAO), Pavia, Italy \\ ${ }^{8}$ Universidade Estadual de Santa Cruz, Ilheus, Brazil \\ E-mail: russo@to.infn.it
}

Received 22 September 2014, revised 3 November 2015 Accepted for publication 10 November 2015

Published 2 December 2015

\section{Abstract}

The calculation algorithm of a modern treatment planning system for ionbeam radiotherapy should ideally be able to deal with different ion species (e.g. protons and carbon ions), to provide relative biological effectiveness (RBE) evaluations and to describe different beam lines. In this work we propose a new approach for ion irradiation outcomes computations, the beamlet superposition (BS) model, which satisfies these requirements.

This model applies and extends the concepts of previous fluence-weighted pencil-beam algorithms to quantities of radiobiological interest other than dose, i.e. RBE- and LET-related quantities. It describes an ion beam through a beam-line specific, weighted superposition of universal beamlets. The universal physical and radiobiological irradiation effect of the beamlets on a representative set of water-like tissues is evaluated once, coupling the pertrack information derived from FLUKA Monte Carlo simulations with the radiobiological effectiveness provided by the microdosimetric kinetic model 
and the local effect model. Thanks to an extension of the superposition concept, the beamlet irradiation action superposition is applicable for the evaluation of dose, RBE and LET distributions. The weight function for the beamlets superposition is derived from the beam phase space density at the patient entrance. A general beam model commissioning procedure is proposed, which has successfully been tested on the CNAO beam line.

The BS model provides the evaluation of different irradiation quantities for different ions, the adaptability permitted by weight functions and the evaluation speed of analitical approaches. Benchmarking plans in simple geometries and clinical plans are shown to demonstrate the model capabilities.

Keywords: beam model, ion therapy, treatment planning, relative biological effectiveness, microdosimetric kinetic model, local effect model, Monte Carlo

(Some figures may appear in colour only in the online journal)

\section{Introduction}

The number of clinical facilities that employ ion therapy to treat cancer is increasing worldwide. Most of these centres feature proton irradiation, since, compared to heavier ions, protons are simpler to handle, and more compact and cheaper accelerators are available on the market. A few centres make use of carbon ions, which are believed to constitute a better option for the treatment of radio-resistant tumours thanks to their favourable increase of the relative biological effectiveness (RBE) in the Bragg peak region. Recently, the use of ions other than proton and carbon ions has been proposed (Kempe et al 2007) and some preliminary assessment of the application of helium and oxygen ion beams has been performed (Fuchs et al 2012, Kurz et al 2012).

The use of ions different from protons entails the use of more complex irradiation-outcome computation algorithms in treatment planning systems (TPS). The reason for this is two-fold: first, the calculation algorithm must account for nuclear fragmentation, which produces a progressive build-up of secondary ions with the depth of penetration in the tissue and hence causes an unwanted dose deposition beyond and aside the Bragg peak; second, it is necessary to evaluate the RBE scaling, which is a patient-specific, spatially-variable and non-linear function of the dose, applying some kind of radiobiological modelling on top of the estimate of the local particle spectra. The RBE actually presents a small level of variability for protons as well (e.g. Gerweck and Kozin 1999, Britten et al 2013, despite the clinical adoption of a constant 1.1 as recommended by the International Commission on Radiation Units and Measurements (ICRU Report 78 2007). The impact of neglecting these RBE variations during treatment planning is under evaluation by several institutions (Tilly et al 2005, Frese et al 2011, Carabe et al 2012, Dasu and Toma-Dasu 2013).

RBE values are usually characterised by big uncertainties (10\% or more) and the employment of different radiobiological models by the different centres hinders the comparability of clinical outcomes (Gueulette and Wambersie 2007, Uzawa et al 2009, Steinsträter et al 2012). For these reasons, it has been proposed that the treatment planning be evaluated solely on the base of physical quantities, using the unrestricted linear energy transfer (the LET $_{\infty}$ defined in the ICRU Report 85a (2011), in this article simply referred to as LET) as a surrogate of the RBE to weight the variations of cell-inactivation efficiency. This approach is 
particularly well-suited to protons, for which a strong correlation between RBE and LET has been observed (Britten et al 2013).

As a consequence of the above, there is growing interest in the ion-therapy community for TPS's capable of dealing with different ion species and of providing a local estimate of the radiobiological effectiveness. Nevertheless, few of the computation algorithms currently employed for clinical use are providing these capabilities. Most of them are in fact tailored to efficiently compute dose distributions after proton irradiation, being analytical or optimised Monte Carlo algorithms that cannot be readily applied to other ions. The most notable exception is TRiP98, a research tool developed and used for planning carbon-ion treatments within the framework of the GSI pilot project in Germany (Krämer et al 2000). This tool has constituted the basis for the implementation of the Syngo ${ }^{\circledR}$ RT Planning software (Siemens Healthcare, Erlangen, Germany), the only commercial TPS to date that incorporates the computation of the RBE for carbon-ion spot scanning. However, the irradiation-outcome computation algorithms of both TRiP98 and Syngo ${ }^{\circledR}$ are not conceived to be easily adapted to different beam lines. For instance, Syngo ${ }^{\circledR}$ requires that the user provides the description of the fragmentation in water by the pencil beams as a part of the commissioning process. The users then have to carry out extensive, centre-specific Monte Carlo simulations in order to provide the TPS with the necessary data.

At INFN we have envisioned an original modelling approach, the beamlet superposition (BS) model, which permits to simulate the irradiation with different ions, to evaluate several physical and radiobiological quantities and to simplify the commissioning procedure. This model has been implemented in a new TPS computing kernel called PlanKIT (planning Kernel for ion therapy), developed in cooperation with IBA. This paper describes the BS model and its application into a treatment planning workflow for spot-scanning (section 2), and provides a demonstration of its capabilities (section 3).

\section{Methods}

\subsection{Principles of the BS model}

The BS model allows computing the three-dimensional effect of an ion field incident on a water-like material. Its present use within the PlanKIT code is to estimate the outcome of a therapeutic ion irradiation delivered through the spot-scanning technique, but the methodology is rather general and could be easily applied to model other problematics related to the interaction of radiation with matter.

The BS model may be considered an extension of previous works on fluence-weighted, elemental-pencil-beam kernels (Schaffner et al 1999, Soukup et al 2005, Kanematsu et al 2009, Fuchs et al 2012). An ion beam - throughout this paper the word 'beam' is used as a synonym of 'spot'-is completely characterised by the phase space distribution of its ions, that is, by the set of positions and momenta owned by its ions at a specific moment in time or while traversing specific surfaces. Every ion beam can be thought of as composed of sub-units, here called beamlets, that are obtained splitting the beam phase space in smaller phase spaces. If the beam is traversing a material, and if the outcome of the interaction of the individual beamlets with the material is known, then the total beam irradiation outcome can be computed summing the separate beamlets action, as long as the considered action is linearly superposable. This problem can be simplified if another point of view is considered. The idea is to start evaluating the average result of the interaction of a very small beamlet with a representative homogeneous material, i.e. water. Then the average action of whatever extended beam on this material could be approximated with a superposition of opportunely positioned and weighted 
replicas of the action of that beamlet. The smaller the adopted beamlet, the better the beam reproduction.

Various physical and radiobiological quantities can be used to describe the local action of an ion beam impinging on a biological tissue (throughout this work, the term local is used to refer to a spatial resolution corresponding to a computed-tomography voxel, that is millimetre scale). The most notable examples currently are:

- The absorbed dose, or simply dose, which is the mean energy imparted to an irradiated volume divided by the mass of that volume (ICRU Report 85a 2011).

- The dose-averaged LET, which is the LET averaged by weighting each LET value by the absorbed dose delivered with a LET between LET and LET + dLET (ICRU Report 16 1970).

- The relative biological effectiveness (RBE), which is the ratio of absorbed dose of a reference beam of photons to the absorbed dose of any other radiation, notably high LET radiations, to produce the same biological effect (ICRU Report 30 1979).

- The RBE-weighted dose, which is the absorbed dose multiplied by the corresponding RBE (ICRU Report 85a 2011).

Since it is possible to express the quantities listed above in terms of linearly-superposable quantities (as shown in section 2.2.2), the BS model allows managing them all in parallel in a comprehensive treatment planning workflow. Throughout the paper, the expression beam irradiation quantities is used for convenience to refer collectively to the spatial distributions of this set of quantities (or their linearly-superposable precursors) that result from a specific beam-tissue interaction.

A schematic view of the BS model workflow is provided in figure 1. Complementing the knowledge about the beamlet irradiation quantities in water with beam-line-specific, irradiation-specific and target-specific information, through the BS model it is possible to derive the beam irradiation quantities in the target. The starting point is the evaluation of the irradiation quantities of a restricted set of small beamlets in water (section 2.2). Since this constitutes a fundamental and universal information, completely decoupled from the specificities of beam lines and targets, it can be evaluated once and stored in look-up tables (LUTs) for later repetitive use. At the TPS commissioning stage, the beam optics is extracted from the experimental data that characterise the considered beam line (section 2.3.3). The beam optics describes how the beam phase space density evolves while the beam is propagating in the treatment room; the related mathematical formulation is reported in sections 2.3.1 and 2.3.2. Knowing the beam optics and the incoming direction of the beam in the target reference system, it is possible to compute the beam phase space density at the target entrance. This phase space density provides the notion of which beamlets to superimpose, how positioned and weighted, in order to reconstruct the beam irradiation quantities. The procedure to perform the roto-translation and weighted superposition of the beamlet irradiation quantities is presented in sections 2.4.1 and 2.4.3. The method to approximate the beam phase space density at the target entrance as a discrete sum of beamlet phase space densities is reported in the appendix. It is important to underline that the same beamlets weights are exploited to determine several physical and radiobiological quantities in parallel, not just the dose; this was not considered in the aforementioned superposition models, which were restricted to dose computations. If the target is not water or is not homogeneous (e.g. a patient), then the beamlets cross different morphologies and produce different results. Therefore, a mapping that associates coordinates in the considered medium to water-equivalent coordinates must be applied to approximately account for the distortion of the beamlet irradiation quantities due to the traversed material (section 2.4.2), as is commonly done in ion therapy (e.g. Schaffner et al 1999, Krämer et al 2000, Soukup 


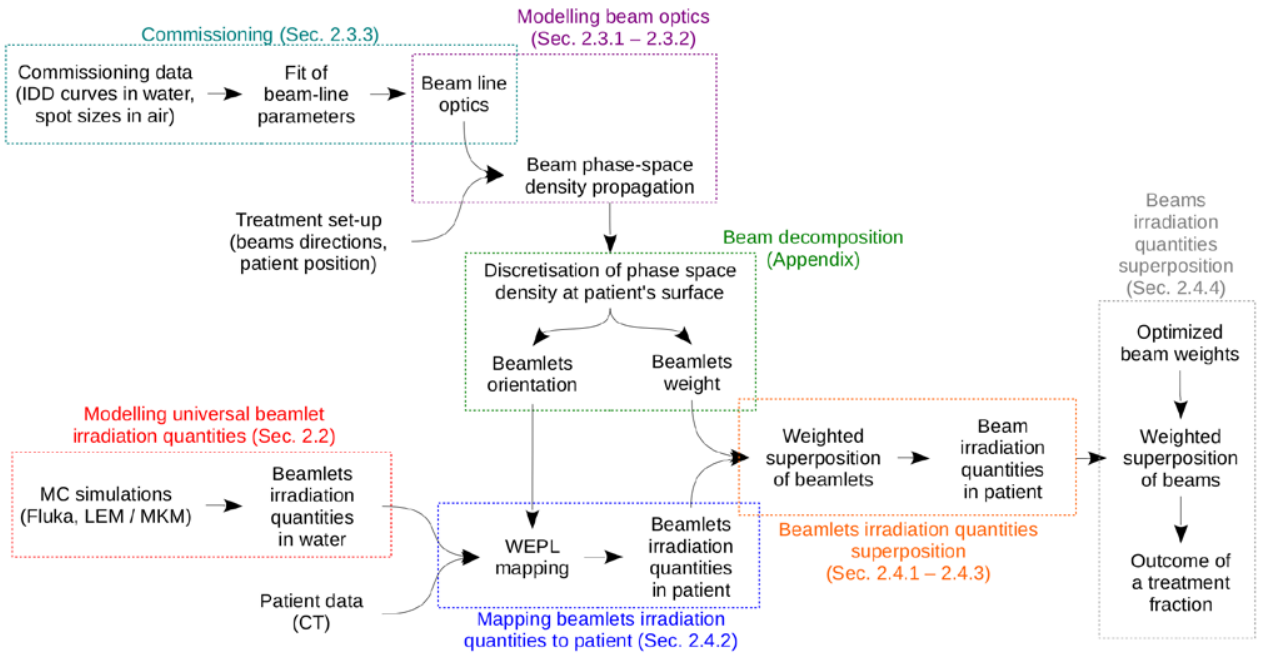

Figure 1. Workflow for a TPS exploiting the BS model.

et al 2005. Once the irradiation action of each of the beams that compose the scanning field has been computed, the total irradiation action can be evaluated (section 2.4.4).

\subsection{Modelling universal beamlet irradiation quantities}

There are several strategies that can be used to describe the interactions of beamlets with matter. In most pencil-beam algorithms (e.g. Krämer et al 2000, Pedroni 2005, Soukup et al 2005, Fuchs et al 2012 the lateral dose envelope is modelled through analytical functions, but this approach requires a lot of fitting effort to fix the functions parameters. In addition, it is very difficult to find a functional form so general that could describe at the same time the irradiation action of different ion species, especially when the irradiation outcome is expressed in terms of different quantities (e.g. dose, LET and RBE). Then we decided to adopt a more flexible approach: the BS model is fed with pre-computed look-up tables (LUTs), containing a threedimensional sampling of the beamlets irradiation quantities; a continuous description is then achieved through interpolation. In this respect, the proposed use of Monte Carlo-generated data resembles what independently proposed by Clasie et al (2012). Since the LUTs are precomputed, the efficiency is not an issue and general purpose Monte Carlo simulation codes can be employed. The fact of exploiting a flexible and widely-employed Monte Carlo simulation code such as FLUKA (Ferrari et al 2005, Battistoni et al 2007) automatically provides the possibility of dealing with different types of primary particles, gives access to detailed physical information, and includes an updated and benchmarked knowledge about fragmentation cross sections.

2.2.1. Monte Carlo simulations of infinitesimal beamlets. The events of injection of single primary ions in a water phantom were simulated using FLUKA. If all the events are generated in the same way, that is, if all the primary ions enter in the phantom in the same position, with the same energy and direction, then the resulting beamlet is called infinitesimal, since it has zero transverse size and no energy dispersion. The infinitesimal beamlet is the smallest and the most general, since its interaction with water corresponds to the average irradiation action 
produced by a single impinging ion, which contains all the information needed to reconstruct the irradiation action of any extended beam in water of the same energy. In reality, since a very large number of infinitesimal beamlets would be required to achieve a smooth superposition, it was preferred to simulate very small, but finite-size beamlets generated with a Gaussian source of $0.2 \mathrm{~mm}$ transverse sigma. These basic beamlets are called quasi-infinitesimal in what follows. The interaction with water of several quasi-infinitesimal beamlets of different energies was simulated, so as to cover the energy range of interest. For protons, the energy ranged from 50 to $250 \mathrm{MeV}$, while for carbon ions it ranged from 60 to $400 \mathrm{MeV} \mathrm{u}^{-1}$. The employed step between energies was 5 and $10 \mathrm{MeV} \mathrm{u}^{-1}$, respectively. In the phantom, about one hundred recording slices were positioned along the beamlet path to extract the relevant tracking information from the simulation. These slices had a thickness comparable to that of a cell nucleus $(10 \mu \mathrm{m})$, and were placed transversely to the beam path. They were more densely located near the Bragg peak, where the dose gradient is higher. In correspondence of each slice the most important characteristics of each track passing through were recorded, such as tracking IDs, position, charge, rest energy, kinetic and deposited energy.

2.2.2. Creating the universal LUTs. The per-track data collected from the simulation was post-processed in order to derive a three-dimensional description of physical quantities such as the dose and the LET, and to apply the radiobiological modelling for the evaluation of RBE-related quantities. The analysis was spatially resolved in the transverse plane by grouping the tracks in radial crowns according to their positions, exploiting the cylindrical symmetry around the beamlet axis. The extracted quantities became part of the LUTs, therefore they ought to be intensive and linearly superposable in order to permit the subsequent linear superpositions. The dose is an intensive and linearly-superposable quantity, while some of the other quantities treated by the present beam irradiation-outcome computation model, such as the RBE or the cell survival probability, are markedly non-linear. However, workarounds to connect these quantities to linearly-superposable entities are possible, as it is shown.

Dose The dose was derived by cumulating the energy depositions from different tracks, separated in slices and radial crowns, and normalising by the mass of the considered volumes and by the number of primary events. The mean dose per primary ion in function of the initial energy and of the position within the phantom was obtained, that is $\langle D\rangle^{0}(E, z, r)$. A visualisation of the dose distribution corresponding to a quasi-infinitesimal beamlet of $270 \mathrm{MeV} \mathrm{u}^{-1}$ carbon ions is shown in figure 2 (top panel). The dose in a point $\vec{r}$ (reference system solidal with the traversed material) related to the composition of $M$ beamlets, each corresponding to a different energy, position and/or direction, is then given by a linear superposition:

$$
\langle D\rangle(\vec{r})=\sum_{i=1}^{M} w_{i}\langle D\rangle_{i}^{0}(\vec{r}),
$$

where $w_{i}$ is given by the number of primary ions belonging to the $i$-th beamlet.

LET-related quantities The quantities of interest are two, the track-averaged LET,

$$
\langle\mathrm{LET}\rangle=\frac{\sum_{j=1}^{N} \mathrm{LET}_{j}}{N},
$$

and the dose-averaged LET, 


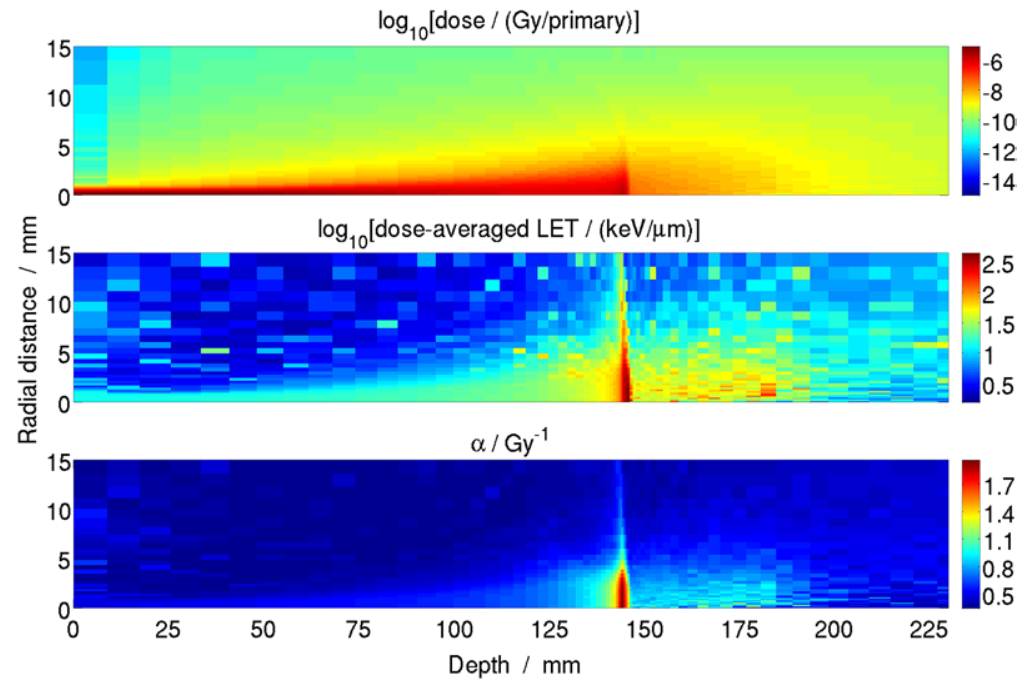

Figure 2. Mean dose per primary ion $\langle D\rangle^{0}(z, r)$, dose-averaged LET $\langle\text { LET }\rangle_{D}(z, r)$ and radiobiological $\alpha(z, r)$ parameter distributions for a quasi-infinitesimal beamlet of $270 \mathrm{MeV} \mathrm{u}^{-1}$ carbon ions impinging on a water phantom. For the evaluation of the $\alpha$ distribution, the MKM parameters reported in Kase et al (2008) for human salivary gland (HSG) cells were used.

$$
\langle\mathrm{LET}\rangle_{D}=\frac{\sum_{j=1}^{N} \mathrm{LET}_{j}^{2}}{\sum_{j=1}^{N} \mathrm{LET}_{j}},
$$

where $N$ is the total number of tracks entering in the considered radial crown through its cross-sectional area $A$. They are not linearly-superposable entities, but can be expressed as combinations of the following linearly-superposable, intensive quantities: the mean track fluence per primary ion,

$$
\left\langle f_{t}\right\rangle^{0}=\frac{N}{N_{p} A},
$$

the mean energy transfer density per primary ion,

$$
\left\langle\rho_{e}\right\rangle^{0}=\frac{\sum_{j=1}^{N} \mathrm{LET}_{j}}{N_{p} A},
$$

and the mean dose-weighted energy transfer density per primary ion,

$$
\left\langle\rho_{e^{2}}\right\rangle^{0}=\frac{\sum_{j=1}^{N} \mathrm{LET}_{j}^{2}}{N_{p} A},
$$

where $N_{p}$ is the total number of simulated primary events. The average LET in a point $\vec{r}$ related to the composition of $M$ beamlets can then be formulated through linear superpositions:

$$
\langle\operatorname{LET}\rangle(\vec{r})=\frac{\sum_{i=1}^{M} w_{i}\left\langle\rho_{e}\right\rangle_{i}^{0}(\vec{r})}{\sum_{i=1}^{M} w_{i}\left\langle f_{t}\right\rangle_{i}^{0}(\vec{r})}
$$




$$
\langle\mathrm{LET}\rangle_{D}(\vec{r})=\frac{\sum_{i=1}^{M} w_{i}\left\langle\rho_{\left.e^{2}\right\rangle_{i}^{0}(\vec{r})}\right.}{\sum_{i=1}^{M} w_{i}\left\langle\rho_{e}\right\rangle_{i}^{0}(\vec{r})} .
$$

Therefore, the $\left\langle f_{t}\right\rangle^{0}(E, z, r),\left\langle\rho_{e}\right\rangle^{0}(E, z, r)$ and $\left\langle\rho_{e^{2}}\right\rangle^{0}(E, z, r)$ quantities were also stored in the LUTs. The $\langle\text { LET }\rangle_{D}$ distribution for a quasi-infinitesimal beamlet of $270 \mathrm{MeV} / \mathrm{u}$ carbon ions is shown in figure 2 (central panel).

Radiobiological quantities The radiobiological quantities provide information about the efficacy of an ion irradiation in inducing specific biological effects. As already discussed, since both the radiation and the tissue quality change across the beam interaction region, a variable local radiobiological effectiveness is expected. The radiobiological effectiveness is described in terms of a local dose-response curve $S(D)$, that gives the probability $S$ for the local tissue to reach a certain end-point, after the tissue has received a dose $D$ through the action of the local ionisation field (it is worth noting that in this paragraph, as everywhere else in the article, the term 'local' should be read as 'at the voxel level'). In this work, as it is common, the considered end-point is the cell survival and the dose-response curve is parametrised according to the linear-quadratic (LQ) model:

$$
S(D)=\mathrm{e}^{-\langle L\rangle}=\mathrm{e}^{-\alpha D-\beta D^{2}} .
$$

The presence of the exponential is commonly motivated by the fact that a cell survives an irradiation in lack of induced lethal events, whose number $L$ is expected to be Poisson distributed. The two parameters, $\alpha$ and $\beta$, which respectively regulate the linear and quadratic dose dependence at the exponent, can be used to concisely describe the doseresponse curve. This description in terms of cell survival is used also when considering functional, non-clonogenic end points. In most of the cases such description is justified, since radiation-produced functional deficits usually result from the depletion of functional cells by cell killing (Hall and Giaccia 2006). In some others an explanation in terms of cell survival is not possible (e.g. nausea, fatigue, somnolence, acute edema, resulting from radiation-induced inflammatory cytokines), and even if the LQ parametrisation is still employed to describe the global dose-effect relationship, its use at the local level as outlined in the present model might not be appropriate.

In the treatment planning with ions, the $\alpha$ and $\beta$ parameters are usually estimated applying radiobiological models. The local effect model (LEM) and the microdosimetric kinetic model (MKM) are so far two of the most employed models. Their detailed explanation is outside the scope of this document; the interested reader is referred to Friedrich et al (2012) and Hawkins (2009) (and citations therein), respectively. These models are able to provide an estimate for $\alpha$ and $\beta$ related to an ion irradiation, starting from the information of the local field composition (the particle spectrum resolved in LET) and the $\alpha_{x}$ and $\beta_{x}$ parameters for conventional x-ray irradiation. Exploiting them, the tracks information recorded from the Monte Carlo simulation was processed to generate threedimensional distributions of $\alpha$ and $\beta$ for the interaction of quasi-infinitesimal beamlets with different tissues. An example of an obtained $\alpha$ parameter distribution is provided in figure 2 (bottom panel).

Unfortunately, neither $\alpha$ nor $\beta$ are linearly superposable. When $M$ irradiation fields are superposed, a well-known rule for the computation of the global LQ parameters is the one proposed by Zaider M and Rossi (1980), which consists of the following dose-weighted averages: 


$$
\begin{aligned}
& \alpha=\frac{\sum_{i=1}^{M} \alpha_{i} D_{i}}{\sum_{i=1}^{M} D_{i}} ; \\
& \sqrt{\beta}=\frac{\sum_{i=1}^{M} \sqrt{\beta_{i}} D_{i}}{\sum_{i=1}^{M} D_{i}} .
\end{aligned}
$$

It can be shown that these summing rules are well in agreement with what is predicted for mixed fields by the LEM and the MKM in the range of applicability of the LQ formalism. Equations (10) and (11) suggest that the sought linearly-superposable quantities are the average number of lethal events per primary depending on $\alpha$,

$$
\left\langle L_{\alpha}\right\rangle^{0}=\alpha\langle D\rangle^{0}
$$

and the square root of the average number of lethal events per primary depending on $\beta$,

$$
\sqrt{\left\langle L_{\beta}\right\rangle^{0}}=\sqrt{\beta}\langle D\rangle^{0} .
$$

In this way the composition in the point $\vec{r}$ of the cell-inactivating effect of $M$ beamlets can be written as an intensity-weighted superposition:

$$
\begin{aligned}
& \left\langle L_{\alpha}\right\rangle(\vec{r})=\sum_{i=1}^{M} w_{i}\left\langle L_{\alpha}\right\rangle_{i}^{0}(\vec{r}) \\
& \sqrt{\left\langle L_{\beta}\right\rangle}(\vec{r})=\sum_{i=1}^{M} w_{i} \sqrt{\left\langle L_{\beta}\right\rangle_{i}^{0}}(\vec{r}),
\end{aligned}
$$

and the quantities $\left\langle L_{\alpha}\right\rangle^{0}(E, z, r)$ and $\sqrt{\left\langle L_{\beta}\right\rangle^{0}}(E, z, r)$ could be included in the LUTs. Other radiobiological quantities such as the survival and the RBE-weighted dose can be easily derived. The survival is simply given by

$$
S=\mathrm{e}^{-\left\langle L_{\alpha}\right\rangle-\left\langle L_{\beta}\right\rangle}
$$

while the RBE-weighted dose $D_{\mathrm{RBE}}$ is just the x-ray dose $D_{x}$ that produces the same survival probability of the specified ion irradiation, that is

$$
D_{\mathrm{RBE}}=\left(D_{x} \mid S_{x}=S\right)=\frac{-\alpha_{x}+\sqrt{\alpha_{x}^{2}+4 \beta_{x}\left(\left\langle L_{\alpha}\right\rangle+\left\langle L_{\beta}\right\rangle\right)}}{2 \beta_{x}} .
$$

It should be kept in mind that the RBE scaling is just one of the conversion factors that contribute to the determination of the iso-effective dose in treatment planning IAEA-TRS461 (2008). However, in this work we describe a method to compute the irradiation effect of single fractions, thus other important dose-equivalence concepts like the biologically effective dose (BED) are not involved.

The described approach is bound to the LQ formalism, but is otherwise not constrained on a specific radiobiological modelling. That is, the LUTs can be filled exploiting a mixture of different radiobiological models and phenomenological findings, provided that their main traits can be formulated in terms of the $\alpha$ and $\beta$ parameters. Unfortunately, the validity of 
the LQ dose-response curve is questioned for high doses per fraction. Indeed, a transition to a purely exponential survival has been observed in several in vitro cell irradiation studies (Andisheh et al 2013), and citations therein). The dose level at which such transition occurs varies depending on the considered cell line, ranging from a few to several tens of Gy. According to what is stated in the review by Shibamoto et al (2012), the LQ model should be applicable up to a radiation dose approximately two-fold the alpha/beta ratio for x-rays. For this reason the proposed method has to be used with caution when assessing the effect of hypo-fractionated treatments. In order to overcome this limitation, the LQ formalism would have to be extended or abandoned in favour of a more general description of the tissue radiation response. For instance, the linear-quadratic-linear (LQL) model could be adopted, following the GSI practice (Kramer and Scholz 2006).

The computed RBE varies not only in virtue of changes in the local particles spectra, but also depending on the biological characteristics of the tissue, the considered end point and the selected radiobiological model. Therefore, representative models of tissue radiation response had to be selected when creating the LUTs, and for each of those different $\left\langle L_{\alpha}\right\rangle^{0}(E, z, r)$ and $\sqrt{\left\langle L_{\beta}\right\rangle^{0}}(E, z, r)$ distributions were generated and tabulated. During treatment planning, in each point of the patient the appropriate radiobiological part of the LUTs is selected as deemed appropriate to emulate the local tissue radiation response. Ideally, there should be a general consensus on the set of tissue models to be considered as representative, in order to facilitate the comparison of the clinical results from different centres, but today the reality is that different modelling approaches are followed by the various institutions. Then the radiobiological part of the LUTs cannot presently be that universal and often need to be recomputed on a per-centre basis, starting from the universal physical properties of the quasi-infinitesimal beamlets. For the scope of this work, the radiobiological modelling adopted by the CNAO centre was implemented, as detailed in section 3.1.

\subsection{Modelling the beam optics of a specific beam line}

The specificities of a beam line are manifested in its beam optics, that is the evolution of the beam phase space around the isocenter, in the absence of a target. We adopted a general modelling, applicable to different beam lines, which extends that proposed in Grevillot et al (2011). The simplest aspects of beam optics theory are applied, adopting the description for a beam composed of identical particles, free of mutual interactions, and propagating in vacuum. The effect produced by the scattering in air around the isocenter is included as an additional average divergence.

Choosing the $Z$ axis as coincident with the beam axis and pointing towards the beam propagation, the time-independent phase space density at a given $z=z^{*}$ plane can be written as $f\left(x, x^{\prime}, y, y^{\prime}, E\right)$, where $x$ and $y$ identify the position on the plane, $x^{\prime}=\mathrm{d} x / \mathrm{d} z$ and $y^{\prime}=\mathrm{d} y / \mathrm{d} z$ the slope of the trajectories and $E$ the energy. If $z^{*}$ is the position at which the entrance face of a water phantom is located, one can exploit the phase space density $f$ as the weighting function in the superposition of an infinite number of infinitesimal beamlets for deriving the composite beam outcome in water (section 2.4). The phase space can be decomposed in two independent phase spaces, one for the energy ( longitudinal phase space) and the other for the spatial attributes, that is positions and directions (transversal phase space). In addition, the spatial part of the phase space can be further split along $X$ and $Y$. We obtain:

$$
\left.f\left(x, x^{\prime}, y, y^{\prime}, E\right)\right|_{z=z^{*}}=\left.\left.g\left(x, x^{\prime}\right)\right|_{z=z^{*}} g\left(y, y^{\prime}\right)\right|_{z=z^{*}} g(E) .
$$


The use of the letter $g$ to designate the single phase-space distributions is not accidental, since in the BS model these probability distributions are assumed to be Gaussian, as it is often the case in beam optics theory. For some beam lines, however, the experimentally-determined phase space density does not resemble a Gaussian distribution; in such cases, the modelling here proposed can still be applied, provided that one first decomposes the non-Gaussian phase space density in the superposition of Gaussian phase space densities, as proposed in Fuchs et al (2012).

2.3.1. The longitudinal phase space. The longitudinal phase space density is the combination of the energy spread coming from the acceleration and energy-selection systems with the energy spread due to the presence of material on the beam path.

When the interaction with passive elements is the main source of longitudinal peak modulation, the representation through an energy spectrum is not the most efficient. A description that considers the probability distribution of the water-equivalent thicknesses (WETs) traversed by the different ions in the beam would be more natural (e.g. Weber and Kraft 1999, Bourhaleb et al 2008, for two reasons:

- The straggling and fragmentation effects occurring in passive materials are more naturally included in that description. In fact, the Bragg peak produced in a water phantom by a beam that has traversed a passive element corresponding to a thickness $\Delta z$ of water-equivalent material can simply be obtained by removing the first $\Delta z$ at the phantom entrance from the Bragg peak that the same beam produces without previous interactions. The reason resides in the very concept of water equivalence. In this way, the shape of the so-obtained peak already includes the effects of the additional straggling and fragmentation. If the passive element corresponds to a distribution of WETs, rather than a single WET, the resulting Bragg peak can be obtained with the weighted superposition of shifted replicas of the non-interacting-beam peak. This can be represented as a mathematical convolution between the WET distribution $w(z)$, reflected about the $y$-axis, and the original Bragg peak shape $d\left(z ; E^{*}\right)$ for a certain impinging beam energy $E^{*}$ :

$$
w(-z) * d\left(z ; E^{*}\right)=\int w\left(-z^{\prime}\right) d\left(z-z^{\prime} ; E^{*}\right) \mathrm{d} z^{\prime} .
$$

The negative sign in $w(-z)$ accounts for the fact that positive WETs correspond to negative shifts.

- The energy spectrum varies depending on the nominal beam energy, while the representation of the passive element with a WET distribution is energy-independent. In this way a single distribution is sufficient to characterize the beam line and can be more easily determined.

Following these motivations, in the BS model the longitudinal phase space description is extended beyond the conventional form, adding a WET distribution. Since the possibility of specifying a spread in energy could still be useful to model the energy dispersion from nonpassive sources, a Gaussian energy term is left in the phase space density. Its interpretation is the following: the pristine Bragg peak to be used in the WET convolution corresponds to a beam impinging on the passive elements that is characterised by a Gaussian energy distribution. The longitudinal phase space density is then modified in $f_{\mathrm{L}}=w(z) \circ g(E)$ and the convolution becomes

$$
w(-z) * d(z ; g(E))=\int w\left(-z^{\prime}\right) d\left(z-z^{\prime} ; g(E)\right) \mathrm{d} z^{\prime} .
$$


The symbol $g$ has not been used for the WET distribution because in general the WETs are not Gaussian distributed. In order to approximate distributions of arbitrary shapes, a discrete probability distribution is used, in the form

$$
w(z)=\sum_{k=1}^{N_{z}} w_{k} \delta\left(z-z_{k}\right) .
$$

2.3.2. The transverse phase space. The following description is restricted to the $\left(x, x^{\prime}\right)$ phase space, the treatment along $\left(y, y^{\prime}\right)$ being independent and identical. According to beam optics theory, the $\left(x, x^{\prime}\right)$ phase space density is described in the BS model by a bivariate Gaussian distribution:

$$
\begin{aligned}
g\left(x, x^{\prime} ; z\right)= & \frac{1}{2 \pi \sigma_{x}(z) \sigma_{x^{\prime}} \sqrt{1-\rho_{x x^{\prime}}^{2}(z)}} \\
& \times \exp \left(-\frac{1}{2\left[1-\rho_{x x^{\prime}}^{2}(z)\right]}\left[\frac{x^{2}}{\sigma_{x}^{2}(z)}-\frac{2 \rho_{x x^{\prime}}(z) x x^{\prime}}{\sigma_{x}(z) \sigma_{x^{\prime}}}+\frac{x^{\prime 2}}{\sigma_{x^{\prime}}^{2}}\right]\right) .
\end{aligned}
$$

In general, the beam is divergent; this makes both the spot width $\sigma_{x}$ and the correlation $\rho_{x x^{\prime}}$ to depend on $z$ according to

$$
\begin{aligned}
& \sigma_{x}(z)=\sqrt{\sigma_{x}^{2}\left(z_{s}\right)+\left(z-z_{s}\right)^{2} \sigma_{x^{\prime}}^{2}} \\
& \rho_{x x^{\prime}}(z)=\frac{\left(z-z_{s}\right) \sigma_{x^{\prime}}}{\sigma_{x}(z)},
\end{aligned}
$$

where $z_{s}$ is the virtual source position, that is the location at which the beam is at its maximum convergence (minimum $\sigma_{x}$ ). In contrast, $\sigma_{x^{\prime}}$ is constant, since we are considering independent particles propagating in vacuum.

2.3.3. Extracting the beam optics from commissioning data. In order to apply the BS model to a specific beam line, the related beam optics must be deduced from the set of measurements performed to characterise the delivery. The usual collection of commissioning measurements, constituted by integral depth-dose profiles in water and 2D spot profiles at different positions in air around the isocenter for a representative set of irradiation configurations, together with some notions about the beam-line design, are sufficient for this purpose. However, also other types of experimental data (e.g. 2D spot profiles at different depths in water) can be exploited, whenever available.

We developed a complex fitting strategy to exploit the commissioning information in a comprehensive way. The accelerating and delivery system is described as a cascade of different beam-modifying components. Each component is characterised by a transfer function that provides the phase-space density transformation experienced by an impinging beam. The sequential application of the transfer functions therefore produces the evolution of the phase space density across the nozzle. The components may correspond to real objects in the beam line, or just be black boxes reproducing in a simplified manner the phenomenology of composite and, possibly, poorly known parts. For instance, the whole accelerating system is usually modelled as a single component transforming an initial infinitesimal phase space density to the phase space density at the end of the vacuum pipe. Other examples of modelling 
components are range shifters and ripple filters. As a matter of fact, most of the passive elements in the beam path, included the beam monitor chambers and the interleaved air layers, can be modelled as equivalent range shifters.

Each transfer function is parametrised in function of some physical attributes of the related component, such as its position, composition, shape, etc. Depending on the amount of available information on the beam line design, each parameter may be fixed or subjected to vary within a more or less restricted range. The parameters that are not fully determined by known constraints are fixed through an optimisation procedure. Starting from a first guess for the free parameters, the phase space density at the nozzle exit is derived through the application of the transfer functions of the present components. Then, the transverse phase space density evolution around the isocenter is computed according to (23) and (24) and compared with the measured behaviour for the spot size in air. At the same time, through the weighted beamlet superposition (section 2.4) the obtained longitudinal phase space density is used to compute the Bragg peak profiles in water, which are also compared with the experimental data. The deviations with respect to the measured data are traduced in a cost function, whose iterative minimisation leads to an optimised choice for the parameters.

All the experimental points are simultaneously fitted. It is especially helpful to dispose of measurements relative to different beam-line set-ups, so to increase the number of mathematical constraints and reduce the degeneracy of the solutions. For instance, taking experimental data in the presence/absence of removable passive elements such as ripple filters provides more information on the permanent components and permits to isolate the contribution of the removable items. Ideally, the derived beam-line description should be physically meaningful and close to the real set-up in order to be trusted when extrapolating to configurations lacking of experimental characterisation. An example of this is the prediction of the beam phase space densities for nominal energies not considered during commissioning.

It is important to underline that the described commissioning procedure, performed on the base of physical quantities such as depth-dose profiles, provides also the information needed to determine the radiobiological beam effectiveness. That is, the derived phase space densities are applicable as weight functions to the superposition of all the linearly-superposable quantities listed in section 2.2.2.

\subsection{Evaluating the beam irradiation quantities}

The computed beam phase space density at the patient's entrance is used as weight for the superposition of the beamlet irradiation actions. For the sake of simplicity, here the superposition is described using integrals, as a continuous sum of an infinite number of infinitesimal beamlets, while in practice a discrete superposition is performed, summing a finite number of quasi-infinitesimal beamlets. All the concepts and formulae related to the discretisation of the beamlets superposition are reported in the appendix. The superposition can be performed either in water, and the resulting beam irradiation quantities mapped to the patient through a single ray-tracing and WEPL-scaling operation (section 2.4.1), or directly in the patient, thereby accounting for the traversed inhomogeneities within the ray-tracing of each beamlet (section 2.4.3). In section 2.4.2 the general WEPL mapping methodology is presented.

2.4.1. Superposing the action of beamlets in water. The objective is to evaluate the irradiation quantities of a beam when it impinges on a water phantom. In order to allow a correspondence with the irradiation action in the patient, the phantom's front face and the patient's entrance must be located at the same $z=z^{*}$ position along the beam line, so to share the same 
incoming beam phase space density. The beam irradiation quantities in water are to be computed combining the beam phase space density at the entrance with the beamlets irradiation quantities pre-evaluated and stored in the LUTs. Each of the linear quantities per primary ion of section 2.2.2 $\left(\langle D\rangle^{0},\left\langle f_{t}\right\rangle^{0},\left\langle\rho_{e}\right\rangle^{0}\right.$, etc...), hereafter generically indicated with $\xi^{0}$, is thus convoluted with the phase space density $f$ at $z=z^{*}$. It is convenient to start applying the longitudinal phase space. If just an energy spread is present, then:

$$
\xi_{\mathrm{L}}^{0}(r, z)=\int g(E) \xi^{0}(E, r, z) \mathrm{d} E .
$$

When also a WET distribution $w(z)$ is present, the previous equation is extended to

$$
\xi_{\mathrm{L}}^{0}(r, z)=\int w\left(-z^{\prime}\right) \int g(E) \xi^{0}\left(E, r, z-z^{\prime}\right) \mathrm{d} E \mathrm{~d} z^{\prime} .
$$

So far, the cylindrical symmetry of the beam has been preserved. Now that we are going to account for the transverse spread, a Cartesian representation must be used in order to deal with possible spot asymmetries. The beam irradiation quantity per primary ion $\xi_{\mathrm{B}}^{0}(\vec{r})$ is evaluated at $\vec{r}=(x, y, z)$ by applying the transverse phase space to $\xi_{\mathrm{L}}^{0}(r, z)$ :

$$
\begin{gathered}
\xi_{\mathrm{B}}^{0}(\vec{r})=\iint g\left(x_{0}, x_{0}^{\prime} ; z^{*}\right) \iint g\left(y_{0}, y_{0}^{\prime} ; z^{*}\right) \xi_{\mathrm{L}}^{0}\left(\left|\vec{u}_{0} \times\left(\vec{r}-\vec{r}_{0}\right)\right|, \vec{u}_{0} \cdot\left(\vec{r}-\vec{r}_{0}\right)\right) \\
\mathrm{d} y_{0} \mathrm{~d} y_{0}^{\prime} \mathrm{d} x_{0} \mathrm{~d} x_{0}^{\prime} .
\end{gathered}
$$

The vectors $\vec{r}_{0}=\left(x_{0}, y_{0}, 0\right)$ and $\vec{u}_{0}=\frac{1}{\sqrt{x_{0}^{\prime 2}+y_{0}^{\prime 2}+1}}\left(x_{0}^{\prime}, y_{0}^{\prime}, 1\right)$ have been used to identify, respectively, the position and orientation of beamlets at the phantom entrance.

The equations (26) and (27) can then be exploited to generate beam-line-specific LUTs. These LUTs contain the beams irradiation quantities in water corresponding to the beamoptics configurations that are relevant for a treatment. Typically, the beam optics depends on the particle type (e.g. protons and carbon ions), the nominal energy, the insertion of passive elements (range shifters, ripple filters) and the position along the beam line. For some beam lines, also changes in the beam optics depending on the magnetic scanning position and on the orientation of the gantry have to be considered. A discrete set of representative configurations is stored in the beam-line-specific LUTs, sampling appropriately the set of possible combinations.

2.4.2. Mapping the beam irradiation quantities to the patient through the WEPL approach. The WEPL approach allows creating a correspondence between the path length of a particle in a heterogeneous material and its equivalent path length in water. The two paths are equivalent in the sense that a particle loses the same average energy while traversing them. By equating the energy loss in the two media, an infinitesimal path length $\mathrm{d} l_{m}$ in a certain material can be mapped to its water-equivalent $\mathrm{d} l_{w}$ :

$$
\mathrm{d} l_{w}=\frac{\rho_{m} S_{m}}{\rho_{w} S_{w}} \mathrm{~d} l_{m}
$$

Here, the definition of mass stopping power $S=\frac{\mathrm{d} E}{\rho \mathrm{d} l}$ has been used. The total water-equivalent path length can then be computed integrating (28), taking into account the variations of $\frac{\rho_{m} S_{m}}{\rho_{w} S_{w}}$ along the particle track. Since the stopping power ratio is weakly dependent on the particle 
type and energy, it can be considered as a local property of the material only. For biological materials this assumption is justified as the error introduced is typically less than $1 \%$.

The BS model uses these concepts to map the beam irradiation quantities in water as contained in the LUTs to the patient geometry. The correspondence is made on the base of the WEPL along the central beam axis, according to the following procedure:

- Ray-tracing operations are performed in the voxelized CT volume to identify the segments of the beam axis belonging to different traversed materials.

- A stopping power ratio is assigned to each CT voxel, exploiting a CT-scanner-specific curve that relates Hounsfield numbers to stopping power ratios.

- WEPLs are attributed to each position along the beam axis, integrating (28).

- For every position $\vec{r}_{m}=\left(x_{m}, y_{m}, z_{m}\right)$ in the beam coordinate system, the projection on the beam axis $z_{m}$ is rescaled to $z_{w}$ according to the WEPL and a water-equivalent position $\vec{r}_{w}=\left(x_{m}, y_{m}, z_{w}\right)$ is obtained.

- The beam irradiation quantity $\xi$ in water is then sampled at $\vec{r}_{w}$ and associated to the considered point $\vec{r}_{m}$ in the patient.

As a result, the beam irradiation quantities taken from the LUTs are longitudinally stretched according to the patient's inhomogeneities along the beam axis. A transverse stretching should also be applied, to account for the variations in multiple scattering occurring in high-density materials, as suggested in Szymanowski and Oelfke (2002). This correction has not yet been implemented in the BS model.

Notice that as a consequence of the coordinate transformation of (28) some of the quantities stored in the LUTs would require a re-normalization in order to preserve the conservation of energy. For instance, for the dose holds:

$$
\frac{D_{m}}{D_{w}}=\frac{\frac{\mathrm{d} E}{\rho_{m} \mathrm{~d} V_{m}}}{\frac{\mathrm{d} E}{\rho_{w} \mathrm{~d} V_{w}}}=\frac{\rho_{w} \mathrm{~d} l_{w}}{\rho_{m} \mathrm{~d} l_{m}}=\frac{S_{m}}{S_{w}} .
$$

However, since it is common practice for TPS's to evaluate the dose-to-water in place of the more realistic dose-to-medium (see the discussion in Paganetti (2009)), this rescaling is not required. This aspect must be taken into consideration when comparing the output of a TPS with that of a Monte Carlo simulation, since the last natively provides the dose-to-medium. In the BS model, these considerations apply as well to the other computed quantities, so the track-averaged-LET-to-water and dose-averaged-LET-to-water are computed, instead of their 'to-medium' counterparts.

2.4.3. Superposing the irradiation action of beamlets in the patient. Clearly, the choice of using the WEPL along the beam axis for the rescaling of the whole beam is a significant approximation. The bigger are the transverse inhomogeneities and the lateral beam extent, the larger is the inaccuracy. In order to improve the treatment of inhomogeneities, it is necessary to employ more ray-tracings per beam.

A natural possibility with the BS model is to exploit the decomposition of the beam in beamlets. Namely, if the superposition of the beamlets irradiation quantities is realised directly in the patient, mapping the quantities of each beamlet from water to tissue according to the WEPL scaling defined along the beamlet axis, each portion of the beam irradiation quantities is deformed according to a more local WEPL and a more faithful representation of the beam propagation is achieved. 
The larger the number of beamlets, the more precise the treatment of heterogeneities is. However, when thousands of beamlets are employed the combination of irradiation quantities becomes too computationally demanding to be evaluated during treatment planning. To limit the number of beamlet components to be traced in the patient to the order of a hundred, finitesize beamlets are to be used instead of the quasi-infinitesimal ones. Therefore, the irradiation quantities in water of a set of small but finite-size beamlets is computed at the BS model commissioning stage, through the superposition of the irradiation quantities of the quasi-infinitesimal beamlets. In the present use, zero-divergence, symmetric beamlets of $0.5-1 \mathrm{~mm}$ sigma are pre-computed. The action of the longitudinal phase space density is included as well in this pre-superposition, since it does not depend upon the presence of heterogeneities.

The status of the implementation of PlanKIT is such that no particular effort has been made so far to optimise computing times. For this reason, the simulation of a clinical treatment plan presently takes some hours when about one hundred beamlets per beam are traced in the patient to better deal with heterogeneities. The computing time can be substantially reduced by parallelising key portions of the code.

2.4.4. Computing the outcome of a single fraction. The procedure described so far deals with the evaluation of the linear irradiation quantities per primary ion produced by single beams, $\xi_{\mathrm{B}}^{0}$. Through linear superposition, each irradiation quantity $\xi_{\mathrm{F}}$ produced by an intensity-modulated ion field (a single treatment fraction) can be computed:

$$
\xi_{\mathrm{F}}(\vec{r})=\sum_{i=1}^{M} \phi_{i} \xi_{\mathrm{B}, i}^{0}(\vec{r})
$$

where $\phi_{i}$ is the intensity (number of primary ions) of the $i$-th beam and $M$ is the number of beams. Then, the linearly-superposable quantities $\left\{\xi_{\mathrm{F}}\right\}$ can be combined as described in section 2.2.2 to derive the non-linearly-superposable quantities (dose-averaged LET, RBEweighted dose, survival).

\section{Results and discussion}

The dose computation capabilities of PlanKIT were benchmarked against experimental data and FLUKA and Syngo ${ }^{\circledR}$ simulations, in order to evaluate the correctness of the implementation and provide some confidence in the BS model usage (sections 3.2-3.6). Five irradiation configurations were considered: single spots, square monoenergetic fields and cubic spreadout Bragg peaks (SOBPs) in homogeneous materials, single beams in a simple heterogeneous geometry and a clinical case. It is worth stressing that it is not part of this work to provide a complete assessment of the clinical usability of the BS model. In addition, in section 3.6.3 some computations are presented to show the kind of analysis the BS model allows. In section 3.1 the operations performed to commission the BS model on the employed beam line are reported.

\subsection{Commissioning the BS model for the CNAO facility}

The BS model has so far been successfully commissioned to the CNAO and WPE (Westdeutsches Protonentherapiezentrum Essen) beam lines. For the sake of brevity, here just the results relative to the horizontal beam line located in the treatment room 3 of CNAO are shown. The CNAO is equipped with a synchrotron and employs quasi-discrete scanning 
(defined in the ICRU Report 78 (2007)), so the amount of material placed on the beam is rather limited. It consists of the vacuum-pipe extraction window, two monitor chambers and about $1 \mathrm{~m}$ of air. When irradiating with carbon ions, two triangular ripple filters of $2 \mathrm{~mm}$ each are added before and after the monitor chambers to achieve an optimal longitudinal beam spread and shape (Giordanengo et al 2015). The accelerator beam extraction line can be configured to provide different spot sizes; so far a single spot size is in use for protons and two for carbon ions.

According to the procedure described in section 2.3.3, the CNAO beam line was modelled as a cascade of simplified beam-modifying components. Several components were identified: a proton accelerator, two carbon-ion accelerators (one for each beam tuning), a ripple filter and a few water-equivalent range shifters (to account for the effect produced by the extraction window, the beam monitors and the surrounding air). The transfer functions of the accelerator components were used to produce the beam phase space density just before the vacuum extraction window. For simplicity such phase space density was considered as originated from a point-like source, constraining $\rho_{x x^{\prime}}=1$. The dependence of the $\sigma_{x}, \sigma_{x^{\prime}}, \sigma_{y}, \sigma_{y^{\prime}}, \mu_{E}$ and $\sigma_{E}$ parameters on the nominal beam energy was described using low order polynomials, in this way imposing a correlation among nominal energies. The action of the components on the longitudinal and transverse phase space densities was separately managed. The longitudinal transfer functions were realised in terms of the WET distribution $w(z)$ of section 2.3.1 and were independent of beam energy, as it is expected from physical considerations. The transverse transfer functions were formulated according to the scattering theory of Highland (1975), which permits to parametrise the increase in spot size and divergence in function of material properties (thickness, density and radiation length) and beam energy. Each of the transfer functions parameters was assigned a limited range of variability, accounting for the known beam-line specifications. The parameters were then fixed fitting experimental data.

As experimental data we exploited the measurements at CNAO for the commissioning of its Syngo ${ }^{\circledR}$ RT Planning installation. This data set consisted of integral depth-dose profiles in water and transverse spot profiles at several positions along the beam direction, for different nominal energies and nominal beam spot sizes, with and without the ripple filters. All data were fitted simultaneously.

In figure 3 a glimpse of the results of the beam model commissioning is provided. The proton and carbon-ion depth-dose curves were fitted with high accuracy, satisfying the $2 \%-0.5 \mathrm{~mm} \gamma$-index criteria everywhere. The derived WET transfer function for a single ripple filter showed roughly evenly-distributed weights in the $0.5-2 \mathrm{~mm}$ range, as expected from a triangular ripple filter of $2 \mathrm{~mm}$ thickness. The found relative energy spread at the vacuum exit window was about $0.1 \%$, a value compliant with the accelerator physicists' estimates. The evolution of proton spot sizes in air was also adequately fitted, with deviations mostly below $3 \%$, except for a few points where differences up to $10 \%$ were obtained. These differences originated both from limitations in the simplified modelling of the beam line and from fluctuations in the experimental determination of the spot sigma (the experimental uncertainties were estimated to be within $0.3 \mathrm{~mm}$ ). With carbon-ion beams, the fit residuals were below $0.3 \mathrm{~mm}$ everywhere. In evaluating these results, one should consider that at CNAO long-term variations of the spot sigma smaller than $10 \%$ and $0.4 \mathrm{~mm}$ are tolerated, according to the considerations of Chanrion et al (2013). Tilted elliptical spot shapes were reported by the CNAO medical physicists for high-energy carbon-ion beams, but lacking a full characterisation of the effect such tilt could not be included in the modelling. The extrapolated proton and carbonion beam phase space densities at the vacuum exit window showed the anticipated decrease of spot size and divergence with increasing beam energy. The beam spread and divergence 

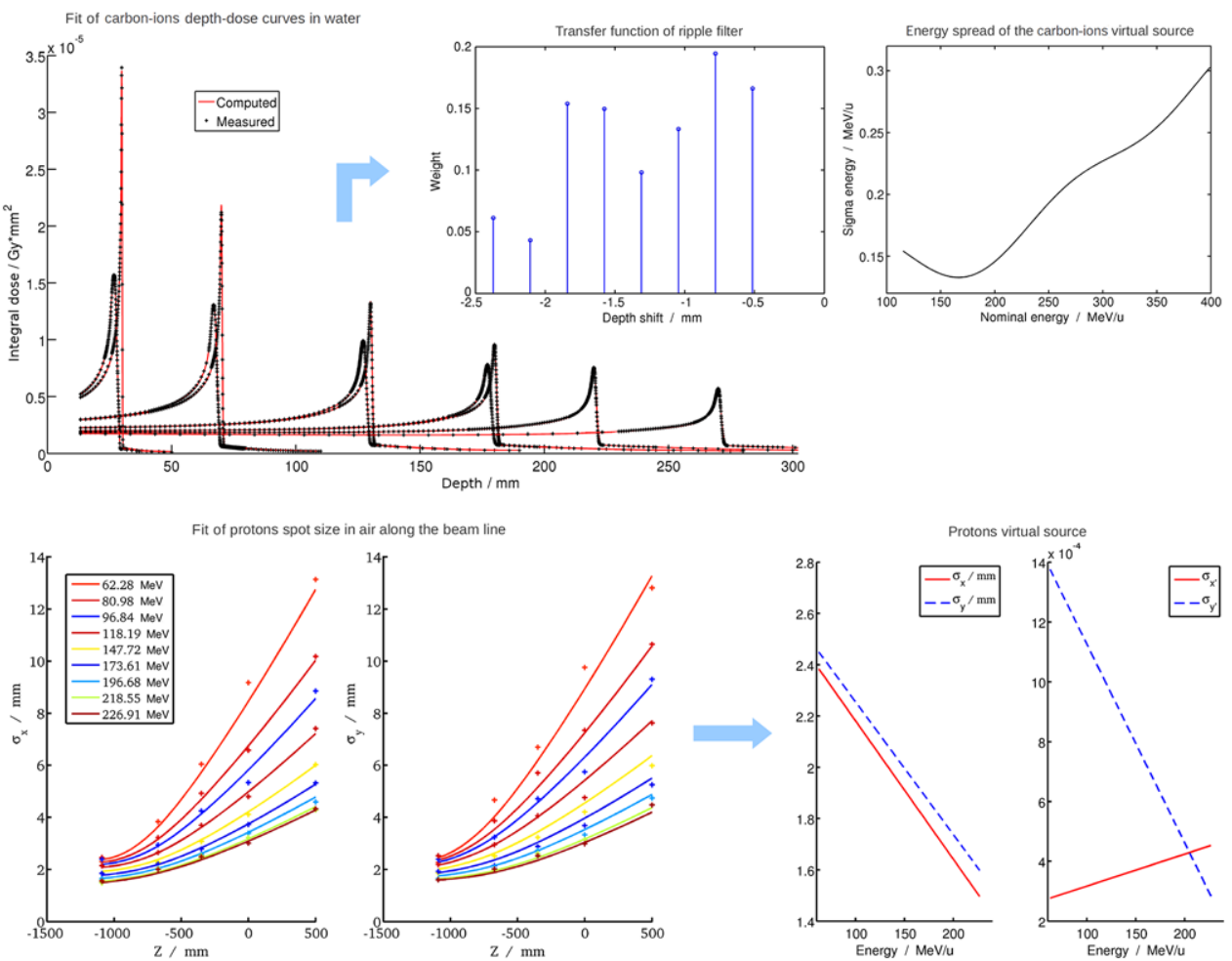

Figure 3. Sample view of the BS model tuning based on the CNAO commissioning data. Upper part: extraction of the ripple-filter $w(z)$ distribution and of the energy spread $\sigma_{E}$ produced by the carbon-ion accelerator through the fit of experimental carbon-ions depth-dose curves in water, in presence and absence of the two ripple filters. Lower part: derivation of the virtual protons source produced by the protons accelerator at the vacuum exit window from the fit of protons spot-size evolution in air.

were found to be more important on the vertical plane ( $y$-axis in figure 3 ) as compared to the horizontal one.

At CNAO, a FLUKA-based simulation tool is in use for research and verification purposes (Tessonnier et al 2014). This tool has been tuned to reproduce the same commissioning data set considered in this work. Its availability enabled us to check the output of PlanKIT against a complete FLUKA simulation (sections 3.5 and 3.6).

As for the radiobiological modelling, the same tissue response made available by Syngo ${ }^{\circledR}$ and used for clinical carbon-ion treatment planning at CNAO and HIT was implemented in the PlanKIT LUTs. It consisted of the brain radiosensitivity predicted by the LEM (version I) using the parameters specified in (Krämer and Scholz 2000).

\subsection{Evaluation of single beams in a homogeneous phantom}

Proton and carbon-ion beam spot profiles were measured after the traversal of different thicknesses of water-equivalent material, in order to check the enlargement of beam size with depth. The measurements were performed using the Lynx ${ }^{\circledR}$ device, a scintillation screen coupled with a CCD camera detector distributed by IBA-Dosimetry (Schwarzenbruck, Germany). This device has a sensible area of $300 \times 300 \mathrm{~mm}^{2}$ and a resolution of $0.5 \mathrm{~mm}$ (Lin et al 2013). 
Originally designed for being used with photon and electron beams, the Lynx ${ }^{\circledR}$ has been characterized and is now extensively being used with proton beams, showing good results. With the help of IBA-Dosimetry, tests were performed at CNAO to check its reliability with carbon-ion beams, obtaining performances comparable to the ones showed with protons. This validation is out of the scope of this paper; related measurements will be referred to in the product data sheet. The Lynx ${ }^{\circledR}$ device was placed on the treatment table behind a water-equivalent solid phantom, realised with variable number of stacked RW3 slabs (PTW-Freiburg, Germany), whose entrance face was kept positioned at the isocenter. Experimental data were collected for the lowest, middle and highest clinical energies $(62.28,147.72$ and $226.91 \mathrm{MeV}$ $\mathrm{u}^{-1}$ for protons, and 115.23, 279.97 and $398.84 \mathrm{MeV} / \mathrm{u}$ for carbon ions), sampling at different positions along the Bragg peak $(0,20,80,140,200,250$ and $300 \mathrm{~mm}$ depth in RW3). The spot intensity profiles were fitted with a two-dimensional Gaussian function in order to extract the spot sigmas along $X$ and $Y$.

For protons, the difference between the spot sigmas measured and simulated by PlanKIT was lower than $5 \%$ and $0.3 \mathrm{~mm}$ in all conditions but at the end of the range with the highest beam energies, where overestimates up to $10 \%$ and $0.7 \mathrm{~mm}$ were observed. Since, as we said previously, at CNAO long-term variations of the spot sigma smaller than $10 \%$ and $0.4 \mathrm{~mm}$ are tolerated and since discrepancies of the order of $10 \%$ were already obtained during the commissioning of the beam model (see section 3.1), such level of disagreement with the measurements can be expected. With carbon ions the measurements confirmed the tilt of the spot profiles at high energies, which was not handled in the commissioning of the BS model. This resulted in a systematic $4-10 \%$ underestimate (overestimate) of the spot width along $x$ (along $y$ ) at middle-high energies. At low energies the agreement was better than $3 \%$.

\subsection{Check of the low-dose contributions}

Proton and carbon-ion scanned fields of different sizes (4, 6, 8, 10, 14 and $19.6 \mathrm{~cm})$ were realised with square grids of equally-weighted spots, for different energies $(62.28,147.72$ and 226.91 $\mathrm{MeV} \mathrm{u}^{-1}$ for protons; 279.97, 342.07 and 398.84 $\mathrm{MeV} \mathrm{u}^{-1}$ for carbon ions). The spot inter-spacing was $2 \mathrm{~mm}$. A water phantom (model 41023, PTW-Freiburg) was placed on the treatment table with its entrance face positioned at the isocenter. The ionization charge was collected at the centre of the fields at different depths (21, 104.8, 209.6 and 275.5 mm) through the use of a Markus chamber (model 34045, PTW-Freiburg) connected to a reference class electrometer (UNIDOS webline, PTW-Freiburg). A correction to account for variations in ambient air temperature and pressure was applied. Three consecutive measurements were performed for each point and the mean value and standard deviation were computed. The standard deviation was globally smaller than $0.5 \%$. Then, field size factors were obtained for both protons and carbon ions by normalising the collected charge by that corresponding to the $10 \times 10 \mathrm{~cm}^{2}$ field. Measuring the dose in the centre of square monoenergetic fields made of evenly spaced spots of equal intensity provides a way to check whether the modelling of the low-dose envelope for a single spot is sufficiently accurate (Sawakuchi et al 2010, Grevillot et al 2011). In fact, the dose measured in the centre of the field increases with the field size due to the contributions coming from the dose halos of the spots added at the field boundary.

The field size factors were estimated by PlanKIT with remarkable accuracy. Concerning the irradiation with proton fields, the percentage difference between the field size factors measured and evaluated by PlanKIT was lower than $1 \%$ for the $78 \%$ of the points, lower than $2 \%$ for $96 \%$ of the points, and lower than $3 \%$ for all the points. With carbon ions the difference was lower than $0.5 \%$ for $93 \%$ of the points and better than $1 \%$ for all the points. This 
Table 1. Configurations considered for the measurement of the cubic SOBPs in the RW3 phantom.

\begin{tabular}{llll}
\hline Particle & SOBP size $\left(\mathrm{cm}^{3}\right)$ & $\begin{array}{l}\text { SOBP range } \\
(\mathrm{mm})\end{array}$ & Depths $(\mathrm{mm})$ \\
\hline $\mathrm{p}$ & $10 \times 10 \times 10$ & $35-135$ & $21.0,84.9,105.8,140.4$ \\
& $10 \times 10 \times 10$ & $135-235$ & $21.0,84.9,140.4,175.0,205.4,241.0$ \\
& $3 \times 3 \times 3$ & $235-265$ & $21.0,84.9,105.8,175.0,241.0,255.7,270.4$ \\
& $5 \times 5 \times 5$ & $265-315$ & $21.0,84.9,140.4,205.4,270.4,295.5$ \\
\hline${ }^{12} \mathrm{C}$ & $6 \times 6 \times 6$ & $60-120$ & $25.2,65.0,95.4,115.3$ \\
& $6 \times 6 \times 6$ & $120-180$ & $25.2,115.3$ \\
& $6 \times 6 \times 6$ & $180-240$ & $25.2,95.4,175.0,214.8,234.8$ \\
\hline
\end{tabular}

Note: All depths and ranges are expressed as water-equivalent.

successful comparison is an indirect confirmation of the validity of the FLUKA description of nuclear interactions, since the universal beamlet quantities in water were derived from FLUKA simulations (section 2.2.2).

\subsection{Evaluation of cubic SOBPs in a homogeneous phantom}

Cubic SOBPs of different sizes and in-depth positions (listed in table 1) were irradiated in RW3 with both proton and carbon ions, with homogeneous RBE-weighted doses of 2 and 3 Gy (RBE), respectively. The measurements were performed placing the Lynx ${ }^{\circledR}$ device on the treatment table behind a variable amount of stacked RW3 slabs, with the entrance face positioned at the isocenter. A flat-field correction was applied to the Lynx ${ }^{\circledR}$ output images in order to compensate for the variation of gain across the sensitive area. Such correction was derived comparing the Lynx ${ }^{\circledR}$ acquisition with the measurement performed with a Gafchromic EBT3 film (International Specialty Products, Wayne, NJ, USA), when delivering a $15 \times 15 \mathrm{~cm}^{2}$ monoenergetic field of uniform fluence in air at the isocenter. The irradiation of the SOBPs was planned using the Syngo ${ }^{\circledR}$ RT Planning (VB10) TPS clinically used at CNAO. The set-up was then simulated with PlanKIT and the resulting physical dose distributions were compared to the measured data. Since the Lynx ${ }^{\circledR}$ output was given in arbitrary units, the integral intensity of each image was normalised to the PlanKIT integral dose at the same depth.

The comparisons over the carbon ion SOBPs were satisfactory, obtaining always more than $98 \%$ of the surface with the $3 \%-3 \mathrm{~mm}$ three-dimensional $\gamma$-index less than 1 . For the proton SOBPs, the dose ripple in positions far from the isocenter were underestimated in the PlanKIT calculation (as shown in figure 4), leading to $91-95 \%$ of the surface with the $3 \%-3 \mathrm{~mm}$ threedimensional $\gamma$-index less than 1 at the distal part of the two deepest SOBPs (for depths in the phantom greater than $23 \mathrm{~cm}$ ). A closer look revealed that the minor ripple was ascribable to a too large computed spot size at high depths. Such observation matches with the discrepancies observed for single spots (section 3.2). In contrast, for the two shallower proton SOBPs, and in general for all depths in the phantom smaller than $23 \mathrm{~cm}$, more than $98 \%$ of the surface was passing the $3 \%-3 \mathrm{~mm} 3 \mathrm{D} \gamma$-index criteria.

The few observed discrepancies can be ascribed (at least partially) to limitations in the BS model commissioning process, and specifically to the use of a commissioning beam data library not tailored for the tuning of the BS model. However, we cannot exclude the eventuality of a concurrent problem in the dose computation algorithm. Further investigations are needed to locate and solve the issue. 


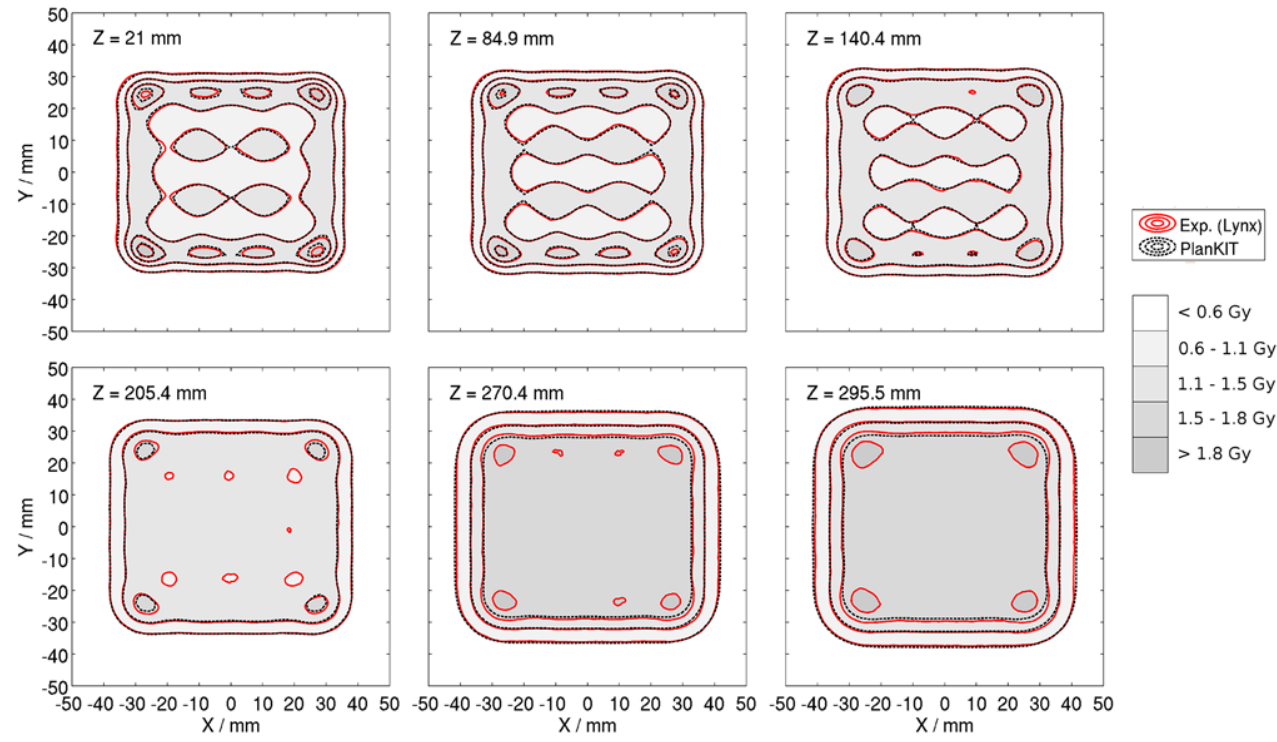

Figure 4. Comparison of measured and PlanKIT-computed transverse dose distributions at six different depths along the deepest irradiated proton SOBP (265-315 $\mathrm{mm}$ range). The drawn isodose lines correspond to the $0.6,0.9,1.2,1.5$ and 1.8 Gy dose levels.

\subsection{Evaluation of single beams in a heterogeneous phantom}

We also tested the BS model performances in the presence of important material heterogeneity. We simulated with FLUKA and PlanKIT a set-up inspired from Soukup et al (2005), which is illustrated in figure 5(a). Monoenergetic beams were directed towards a heterogeneous phantom consisting of four adjacent homogeneous blocks made of different materials. The beams were first traversing a slab of $1 \mathrm{~cm}$ depth skin tissue $(\mathrm{HU}=74)$, then passed through a composite slab of $1 \mathrm{~cm}$ depth made up of bone $(\mathrm{HU}=1524)$ on one side and of lung $(\mathrm{HU}=-741)$ on the other, and finally crossed an extended region of soft tissue $(\mathrm{HU}=0)$. The dose-to-tissue computed by FLUKA was converted to dose-to-water in order to perform a meaningful comparison with the dose-to-water provided by PlanKIT, as discussed in section 2.4.2.

Figure 5 reports an extract of the results of the comparison. Few minor differences were observed. First, a small overestimation of the beam range was found for all energies (at most $1 \mathrm{~mm}$ difference at high energy). This is likely arising from small differences between the conversion from Hounsfield numbers to WEPL implemented in PlanKIT and the one from Hounsfield numbers to material properties set in Fluka. In any case, this discrepancy was found to be of limited relevance when simulating clinical conditions (as shown in section 3.6.2). In addition, since beamlets of $0.5 \mathrm{~mm}$ sigma were employed in the superposition, the dose distributions produced by PlanKIT were slightly smeared; this led to visible discrepancies in the high-gradient region between the two peaks, where a different extent of the lateral dose fall-offs was observed. All in all, PlanKIT reproduced fairly well the beam perturbation caused by the presence of the material discontinuity, with results comparable to those reported in similar studies (Soukup et al 2005, Grevillot et al 2012).

\subsection{Evaluation of a treatment plan}

In order to assess the BS model accuracy in clinical conditions, we simulated with PlanKIT the irradiation of a clival chordoma, exploiting the DICOM data set of a patient treated at CNAO. 


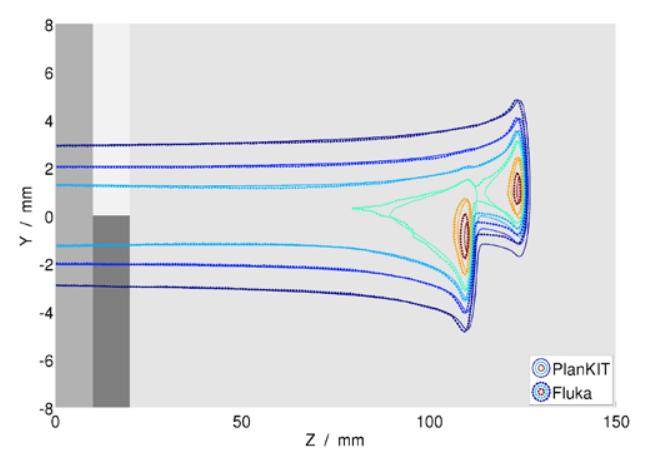

(a)

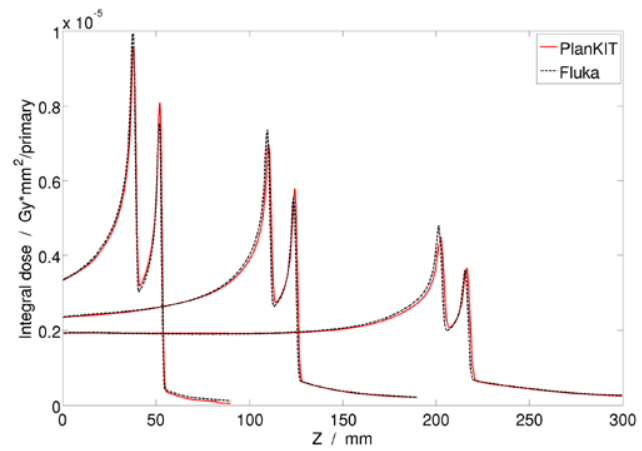

(b)

Figure 5. Comparison of FLUKA and PlanKIT dose-to-water distributions for carbonion beams hitting a lateral heterogeneity. (a) Isodose lines in the central plane of the $250 \mathrm{MeV} \mathrm{u}^{-1}$ beam. (b) Integrated depth-dose profiles for 150,250 and $350 \mathrm{MeV} \mathrm{u}^{-1}$ beams.

3.6.1. Comparison with Syngo ${ }^{\circledR}$. The actual clinical treatment was planned at CNAO with the Syngo ${ }^{\circledR}$ software, using two laterally-opposed carbon-ion beams. As it is done routinely, the radiosensitivity of brain tissue was attributed to the whole head, using the LEM I with parameters specified in Krämer and Scholz (2000). According to the clinical protocol for skull-base chordoma, an RBE-weighted dose of 70.4 Gy (RBE) divided in 16 fractions of 4.4 Gy (RBE) each was prescribed to the target volume. The main irradiation constraints specified for this particular case were a maximum RBE-weighted dose of $30 \mathrm{~Gy}$ (RBE) to the brain stem and a maximum RBE-weighted dose of $60 \mathrm{~Gy}(\mathrm{RBE})$ to the pharynx. The RBE-weighted dose to the parotid glands was kept as low as possible without compromising the target coverage.

A single fraction of the delivered treatment was simulated with PlanKIT, and the produced physical and RBE-weighted dose distributions were compared with those reported by Syngo ${ }^{\circledR}$ (figure 6). The two physical dose distributions were found to be remarkably similar, with the $3 \%-3 \mathrm{~mm} \gamma$-index criteria satisfied everywhere. On the other hand, the corresponding RBE-weighted dose distributions differed sensibly, since the RBE-weighted dose predicted by PlanKIT was found to be everywhere $2-3 \%$ lower than that estimated by Syngo ${ }^{\circledR}$. The observed difference in RBE scaling may result from either a different modelling of fragmentation or a slightly different implementation of LEM I, or both. However important this discrepancy might seem, it did not prevent the RBE-weighted dose distribution to pass the $3 \%-3 \mathrm{~mm}$ $\gamma$-index criteria in more than $98 \%$ of the PTV and in more than $99 \%$ of the patient volume having a dose greater than $1 \%$ of the maximum.

3.6.2. Comparison with FLUKA. As an additional test, we planned from scratch with PlanKIT a carbon-ion treatment using the CT images and structures of the same clinical study of section 3.6.1, and we checked the resulting physical dose distribution against a FLUKA simulation. We kept the clinical set-up of two laterally opposed fields, while we altered the prescription, requesting the delivery of a homogeneous RBE-weighted dose of $3 \mathrm{~Gy}$ (RBE) to a smaller PTV (target of the boost irradiation fractions) and avoiding the setting of constraints on organs at risk, in order to simplify the comparison. Also in this case the LEM I brain tissue radiosensitivity was adopted. The two fields were concurrently optimised with PlanKIT, using the intensity modulated particle therapy (IMPT) technique, as defined in Lomax (1999). The optimised spot intensities were then provided as input to FLUKA for the computation of 

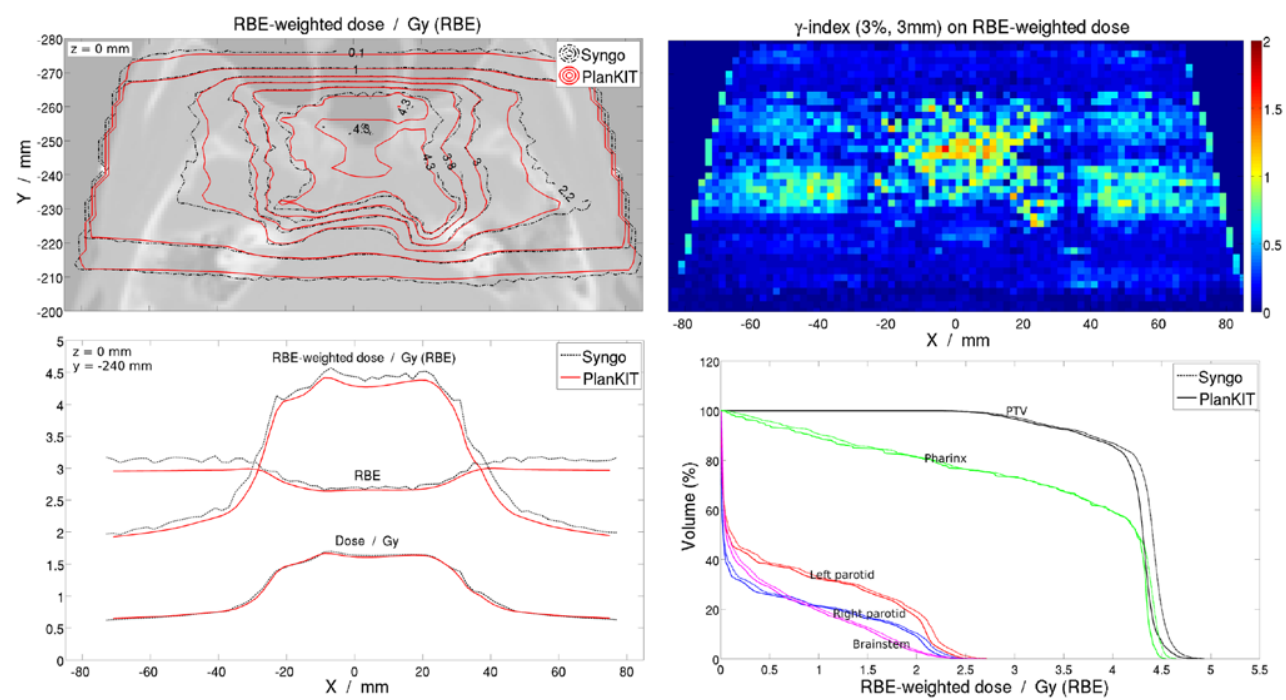

Figure 6. Comparison between PlanKIT- and Syngo ${ }^{\circledR}{ }^{-}$-generated dose distributions corresponding to the irradiation of a skull-base chordoma with two opposing carbonion fields.

the physical dose. A scoring-grid resolution of $2 \times 2 \times 2 \mathrm{~mm}^{3}$ was employed in PlanKIT and Fluka.

A view of the obtained results is presented in figure 7. Differences between the physical dose distributions produced by PlanKIT and FLUKA were visible both in the entrance path of the fields and in the PTV. However, these discrepancies are tolerable since the 3\%-3 mm $\gamma$-index criteria was satisfied in $95.5 \%$ of the PTV and in more than $99 \%$ of the patient volume having a dose greater than $1 \%$ of the maximum. Most of the differences in the PTV arose from a $1 \mathrm{~mm}$ range overestimation by PlanKIT for each field, in conformity to what reported for single beams (section 3.5). A good agreement between the RBE scaling evaluated by the two tools was found, except at the patient entrance and at the center of the PTV where discrepancies up to $2 \%$ were observed. Considering the RBE-weighted dose, the $3 \%-3 \mathrm{~mm} \gamma$-index criteria was satisfied in $95.9 \%$ of the PTV and in more than $99 \%$ of the patient volume having a dose greater than $1 \%$ of the maximum. The deviations observed for the RBE-weighted dose were present mainly in consequence of the physical dose differences.

3.6.3. PlanKIT analysis. In order to illustrate the possibilities offered by the BS model, a side-by-side evaluation of RBE and dose-averaged LET distributions following proton and carbon-ion irradiation is presented in figure 8 for the same treatment set-up considered in section 3.6.2. The same beams set-up and prescription described above were applied. To exploit the BS model prerogatives, a variable three-dimensional RBE scaling was considered also for the proton case, instead of applying a constant 1.1 factor. Since the LEM I is admittedly not well-suited to describe the radiobiological effectiveness of protons (Elsässer and Scholz 2007), we chose to employ the MKM instead, adopting the model parameters used in Kase et al (2008) to reproduce the in vitro response of human salivary gland (HSG) cells.

The RBE modulations observed on the irradiation axis were comparable to those reported in previous studies (Elsässer et al 2010, Böhlen et al 2012). For protons the RBE increased from 1.04 at the entrance to 1.1-1.2 in and around the PTV; for carbon ions the modulation 

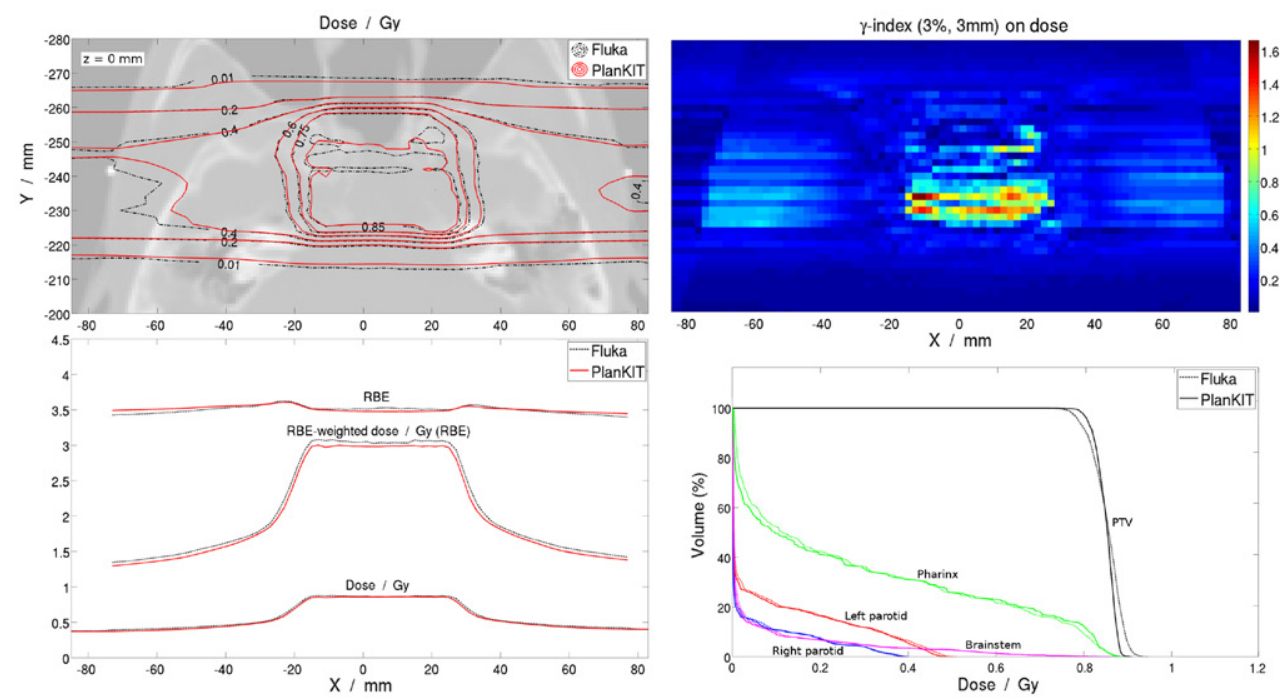

Figure 7. Comparison between PlanKIT- and FLUKA-generated dose distributions corresponding to the irradiation of a skull-base chordoma with two opposing carbonion fields.

was more pronounced, with values ranging from 1.5 at the patient entrance to 2.3 in the PTV.

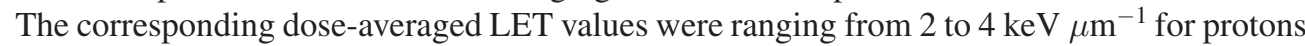
and from 15 to $80 \mathrm{keV} \mu \mathrm{m}^{-1}$ for carbon ions. Thanks to the 3D per-beam irradiation outcome description provided by the BS model, also the lateral RBE and dose-averaged LET modulation could be inspected. The simulation evidenced a rarely shown and hence poorly known fact, that a noticeable RBE increase exists in correspondence of the lateral SOBP penumbra. The reason for this increase is twofold: first, as it can be seen from the corresponding doseaveraged LET distributions, the small ion population that reaches these regions is relatively richer in high-LET components; secondly, the RBE has a marked inverse dependence over dose. Values of RBE as high as 1.5 and 4 were obtained for protons and carbon ions, respectively, in the transverse dose penumbra. Since the dose there was rather low, these high RBE values would have been of small clinical concern; they just produced a slight extension of the lateral penumbra, in a way that resembles the more investigated biologically-effective proton range shift (Carabe et al 2012).

\section{Conclusions}

An algorithm for the computation of the physical and biological irradiation action of ion beams, the BS model, has been proposed and successfully implemented. It applies and extends the concepts of previous fluence-weighted pencil-beam algorithms to quantities of radiobiological interest other than dose, e.g. RBE- and LET-related quantities. Since the beamlet irradiation quantities in water are derived from Monte Carlo simulations, the obtained three-dimensional description is more detailed and more flexible compared to the usual analytical approaches: it allows expressing the outcome of the irradiation of different ion species in terms of different quantities, within the same framework.

The BS model also extends the treatment of beam optics. Namely, it provides a description for the transverse phase-space density which accounts for the beam divergence and 

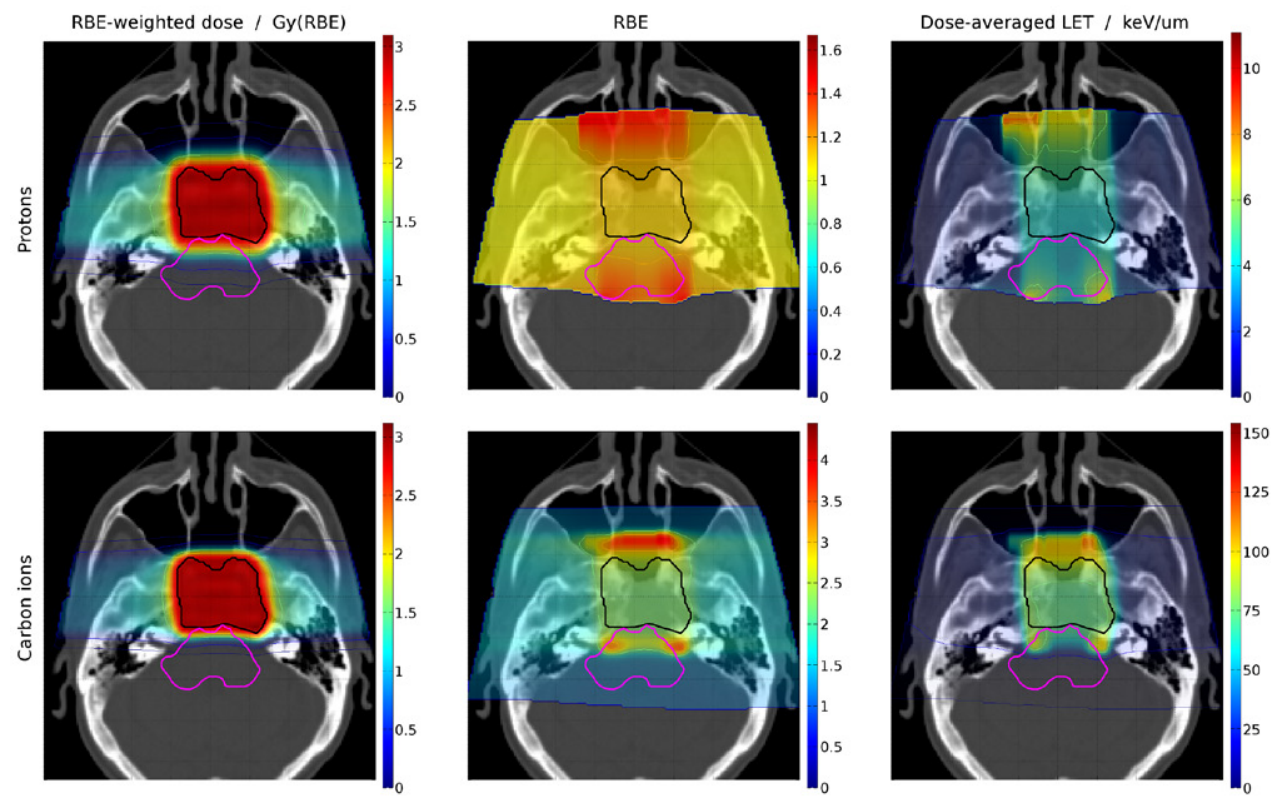

Figure 8. Transverse view of RBE-weighted dose, RBE and dose-averaged LET distributions, obtained planning with PlanKIT the irradiation of a head \& neck tumour with two opposing proton or carbon-ion fields. The black line encloses the PTV, while the magenta line delineates the brain stem.

emittance, and employs a description for the longitudinal phase-space density based on WET distributions that is inherently well-suited to model the action of passive beam line elements. Furthermore, a general commissioning procedure has been devised in order to extract the beam optics parameters and easily tune the BS model on a specific beam line. Thanks to the extension of the superposition concept to all the radiobiological quantities, the beam optics derived from physical experimental data can be applied as a weight function for the derivation via superposition of all the radiobiological quantities. This approach eliminates the need for extensive, ad-hoc Monte Carlo simulations for the derivation of the LET spectra and of the resulting RBE, as it is required for the commissioning of Syngo ${ }^{\circledR}$.

The BS model has been implemented in the PlanKIT software and several tests were run to check its accuracy. The comparisons with experimental data and with FLUKA and Syngo ${ }^{\circledR}$ simulations provide high confidence in the model usage, albeit they cannot be considered sufficient to fully validate it for clinical applications. That is, the seeming overestimation of lateral spot size in water at high energies reported in sections 3.2 and 3.4, albeit small, cannot be overlooked. Besides, the radiobiological modelling must be subjected to further checks, benchmarking the use of LEM in the BS model either against TRiP98 or against in vitro experimental data, along the lines of what done by Krämer et al (2003). The implementation of the MKM in the BS model must be verified as well. However, we can already state that the BS model provides a flexible way to perform advanced radiobiological computations with close-to-clinical accuracy. As such, it can be exploited to perform comparative studies over the use of different ion species, radiobiological models and protocols. 


\section{Acknowledgments}

This work has been conducted in the framework of a cooperation agreement between the Istituto Nazionale di Fisica Nucleare (INFN), Italy, and the Ion Beam Applications (IBA) company, Belgium, through their joint financial support. The authors would like to thank the CNAO staff for the technical support provided during the data taking.

\section{Appendix. Discrete beamlet superposition formulae}

The implementation of the BS model in a computer requires that a discrete formulation of the concepts reported in section 2.4 is employed. That is, the beam phase space density at the patient's entrance must be discretised in order to be used as a weight for the beamlet superposition in water. The purpose is to find a good phase-space density decomposition that would allow obtaining the correct evaluation of the beam irradiation quantities (within a few percents) superposing a manageable number of beamlets. This task is facilitated by the sensible choice made for the description of the phase space density: except for the WET part of the longitudinal phase space density, which is already discretised, all the employed probability distributions are Gaussians.

\section{A.1. Discretisation of a Gaussian distribution}

A simple way of splitting a Gaussian distribution

$$
g\left(r ; \mu_{r}, \sigma_{r}\right)=\frac{1}{\sigma_{r} \sqrt{2 \pi}} \exp \left(-\frac{\left(r-\mu_{r}\right)^{2}}{2 \sigma_{r}^{2}}\right),
$$

having mean $\mu_{r}$ and standard deviation $\sigma_{r}$, is that of approximating it with a weighted superposition of $N$ smaller Gaussian distributions $g\left(r ; 0, \tilde{\sigma}_{r}\right)$. If the superposition was made of infinite Gaussian components, infinitesimally-spaced between $-\infty$ and $+\infty$, then it could be written in the form of a convolution, that is

$$
g\left(r ; \mu_{r}, \sigma_{r}\right)=g\left(r ; \mu_{r}, \hat{\sigma}_{r}\right) * g\left(r ; 0, \tilde{\sigma}_{r}\right),
$$

using as weight function the Gaussian distribution $g\left(r ; \mu_{r}, \hat{\sigma}_{r}\right)$ whose standard deviation $\hat{\sigma}_{r}$ would be obtained subtracting in quadrature $\tilde{\sigma}_{r}$ from $\sigma_{r}$ :

$$
\hat{\sigma}_{r}=\sqrt{\sigma_{r}^{2}-\tilde{\sigma}_{r}^{2}} .
$$

But in practice the superposition must be made up of a finite number of terms. The approximation of the Gaussian weight function with a train of $N$ Dirac's deltas, equally-spaced by an opportune $\Delta r$ between $-3.5 \hat{\sigma}_{r}$ and $+3.5 \hat{\sigma}_{r}$,

$$
g\left(r ; \mu_{r}, \hat{\sigma}_{r}\right) \simeq \sum_{i=-\frac{N-1}{2}}^{+\frac{N-1}{2}} g_{r, i} \delta\left(r-\mu_{r}-i \Delta r\right),
$$

provides the sought discretisation:

$$
g\left(r ; \mu_{r}, \sigma_{r}\right) \simeq \sum_{i=-\frac{N-1}{2}}^{+\frac{N-1}{2}} g_{r, i} g\left(r ; \mu_{r}+i \Delta r, \tilde{\sigma}_{r}\right) .
$$


The weights $g_{r, i}$ are computed integrating the area underneath the $g\left(r ; \mu_{r}, \hat{\sigma}_{r}\right)$ Gaussian weight curve in a range of $\pm \frac{\Delta r}{2}$ around each of the beamlet central positions:

$$
g_{r, i}=\int_{\mu_{r}+\left(i-\frac{1}{2}\right) \Delta r}^{\mu_{r}+\left(i+\frac{1}{2}\right) \Delta r} g\left(r ; \mu_{r}, \hat{\sigma}_{r}\right) \mathrm{d} r
$$

Sometimes it is not necessary for the discretisation to closely reproduce the original Gaussian profile. In such cases a decomposition in a train of Dirac's deltas as the one in (A.4) may be sufficient.

\section{A.2. Discretisation of the longitudinal phase space density}

The Gaussian energy distribution $g\left(E ; \mu_{E}, \sigma_{E}\right)$ is approximated with a weighted sum of $N_{E}$ monoenergetic beamlets, equispaced in energy between $\mu_{E}-3 \sigma_{E}$ and $\mu_{E}+3 \sigma_{E}$. The relative longitudinal phase space density can be expressed using the notation introduced in section A.1:

$$
g\left(E ; \mu_{E}, \sigma_{E}\right) \simeq \sum_{l=-\frac{N_{E}-1}{2}}^{+\frac{N_{E}-1}{2}} g_{E, l} \delta\left(E-\left(\mu_{E}+l \Delta E\right)\right) .
$$

The spacing $\Delta E$ must be chosen in order to provide a good superposition of the irradiation outcomes of the beamlets, that is, to avoid that the discrete structure of the approximated phase space density is manifested in the composite irradiation outcome. Setting $\Delta E=0.1 \mathrm{MeV} / \mathrm{u}$ is usually appropriate. Introducing (A.7) in the energy superposition of (25), we obtain:

$$
\xi_{\mathrm{L}}^{0}(r, z) \simeq \sum_{l=-\frac{N_{E}-1}{2}}^{+\frac{N_{E}-1}{2}} g_{E, l} \xi^{0}\left(\mu_{E}+l \Delta E, r, z\right)
$$

Then we need to consider the WET distribution $w(z)$. The WET distribution of section 2.3.1 is already defined as a discrete set of weights, therefore no further decomposition is required. The present choice is to use $N_{z}$ equispaced beamlet shifts:

$$
w(z)=\sum_{k=0}^{N_{z}-1} w_{k} \delta(z-k \Delta z) .
$$

The spacing $\Delta z$ must be chosen sufficiently small in order to guarantee a good superposition of the shifted beamlets. $\Delta z$ is usually chosen between 0.1 and $1 \mathrm{~mm}$.

The employment of (A.7) and (A.9) in the longitudinal superposition of (26) gives:

$$
\xi_{\mathrm{L}}^{0}(r, z) \simeq \sum_{k=0}^{N_{z}} w_{k} \sum_{l=-\frac{N_{E}-1}{2}}^{+\frac{N_{E}-1}{2}} g_{E, l} \xi^{0}\left(\mu_{E}+l \Delta E, r, z+k \Delta z\right)
$$

\section{A.3. Discretisation of the transverse phase space density}

This section deals with the task of decomposing bivariate, correlated Gaussian distributions in the $\left(x, x^{\prime}\right)$ space, taking into account their evolution during the beam propagation along $z$. An analogous treatment holds for the $\left(y, y^{\prime}\right)$ space. 
Substituting (23) and (24) into (22), and performing some algebraic manipulations, we change the representation of the transverse phase space density:

$$
\begin{aligned}
g\left(x, x^{\prime} ; z\right) & =\frac{1}{2 \pi \sigma_{x}\left(z_{s}\right) \sigma_{x^{\prime}}} \exp \left(-\frac{\left[x-\left(z-z_{s}\right) x^{\prime}\right]^{2}}{2 \sigma_{x}^{2}\left(z_{s}\right)}\right) \exp \left(-\frac{x^{\prime 2}}{2 \sigma_{x^{\prime}}^{2}}\right) \\
& =g\left(x ; \mu_{x}\left(z, x^{\prime}\right), \sigma_{x}\left(z_{s}\right)\right) \cdot g\left(x^{\prime} ; \sigma_{x^{\prime}}\right) .
\end{aligned}
$$

This way of writing explicitly shows that the marginal Gaussian distribution along $X^{\prime}$ remains constant during the beam propagation. Also, it reveals that the phase space density profiles along $X$ for fixed $x^{\prime}$ values are Gaussian distributions all having the same constant $\sigma_{x}\left(z_{s}\right)$ standard deviation; during the beam propagation they shift along $X$ of different amounts, according to their respective $x^{\prime}$ position and to the $\mu_{x}=\left(z-z_{s}\right) x^{\prime}$ law, as it is shown in figure A1.

It is then possible to proceed with the discretisation. The main objective is not a good reproduction of the phase space density, rather a precise description of the beam irradiation outcome in the patient. However, the fluence distribution is strongly connected to the dose distribution (especially at the patient entrance, where the scattering in the medium has just taken place), so it is necessary that the projection of the transverse phase space density on the $x$ axis is not significantly altered by the discretisation. On the other hand, the details of the distribution along $X^{\prime}$ do not influence directly the spatial distributions, but only indirectly through the $x-x^{\prime}$ correlation. Following these considerations, the discretisation along $X^{\prime}$ can be performed without too much concern, approximating the Gaussian $g\left(x^{\prime} ; 0, \sigma_{x^{\prime}}\right)$ distribution with a weighted sum of $N_{x^{\prime}}$ Dirac's deltas, equispaced along $x^{\prime}$ between $-3.5 \sigma_{x^{\prime}}$ and $+3.5 \sigma_{x^{\prime}}$ (figure A1):

$$
g\left(x^{\prime} ; 0, \sigma_{x^{\prime}}\right) \simeq \sum_{i^{\prime}=-\frac{N_{x^{\prime}}-1}{2}}^{+\frac{N_{x^{\prime}}-1}{2}} g_{x^{\prime}, i^{\prime}} \delta\left(x^{\prime}-i^{\prime} \Delta x^{\prime}\right) .
$$

Adopting this approximation in (A.12), the transverse phase space density becomes

$$
g\left(x, x^{\prime} ; z\right) \simeq \sum_{i^{\prime}=-\frac{N_{x^{\prime}}-1}{2}}^{+\frac{N_{x^{\prime}}-1}{2}} g_{x^{\prime}, i^{\prime}} \delta\left(x^{\prime}-i^{\prime} \Delta x^{\prime}\right) g\left(x ; \mu_{x}=\left(z-z_{s}\right) x^{\prime}, \sigma_{x}\left(z_{s}\right)\right) .
$$

In this way the beam is modelled as the superposition of $N_{x^{\prime}}$ identical and zero-divergence beamlets of $\sigma_{x}\left(z_{s}\right)$ spot size, opportunely oriented and weighted. A certain care must be used in choosing the $\Delta x^{\prime}$ spacing, in order to ensure that in the point of maximum transverse spread within the patient the discretisation along $X^{\prime}$ is not producing visible effects on the $X$ projection. This is achieved if in the range of interest along $Z$ (which generally corresponds to the space between the patient's entrance and the Bragg peak position) the $N_{x^{\prime}}$ Gaussian profiles of (A.14), which are moving along $x$ at different $i^{\prime} \Delta x^{\prime}$ rates, never get apart more than a certain fraction of their sigma.

It is now possible to tackle the discretisation along $X$. Each of the identical Gaussian distributions in (A.14) must be split into the sum of $N_{x}$ components. In this case is not possible to employ the previously-adopted approximation of a Gaussian through a train of Dirac's deltas, because otherwise the discretisation would be manifested, at least at the patient entrance. 


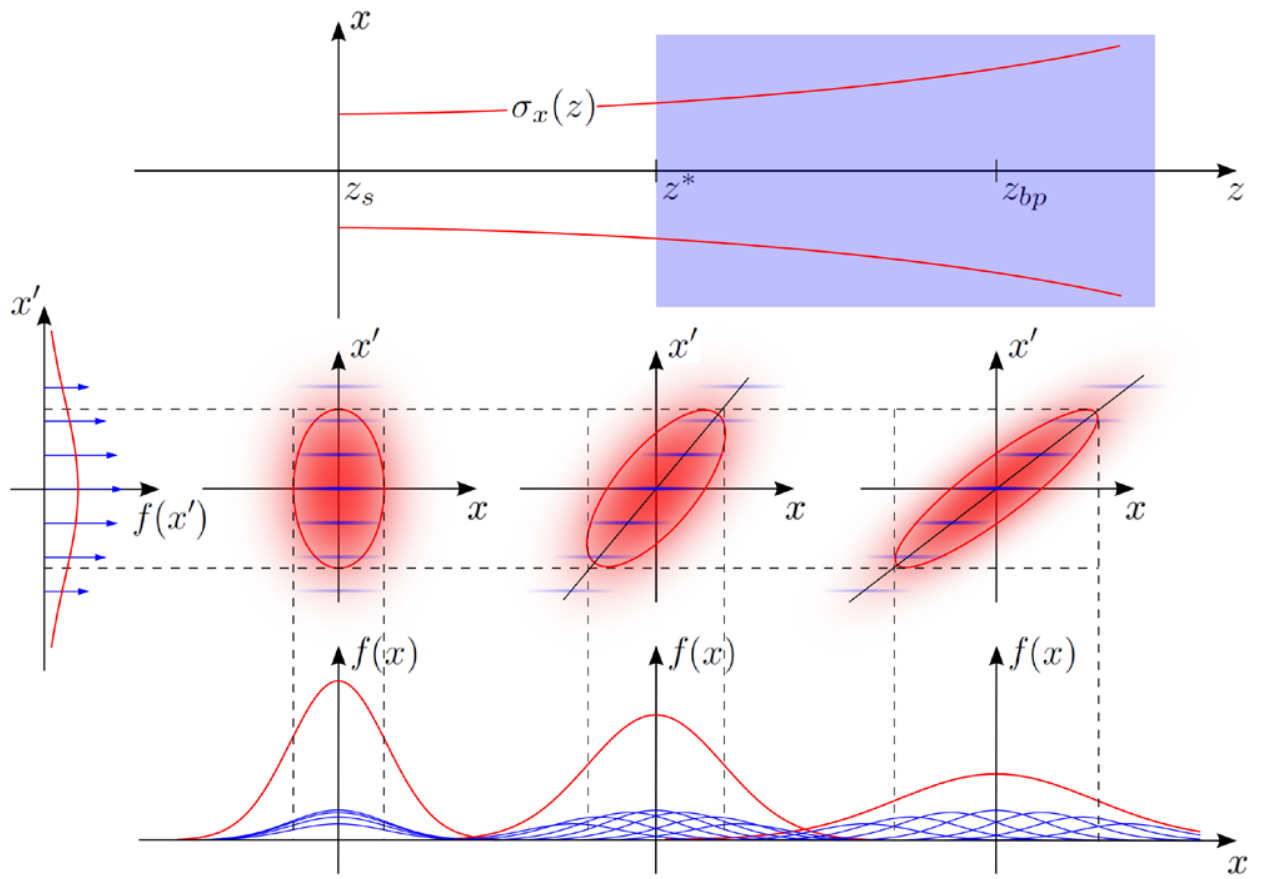

Figure A1. Visualisation of the transversal phase-space density decomposition through the approximation of the $g\left(x^{\prime} ; 0, \sigma_{x^{\prime}}\right)$ distribution with a weighted sum of equispaced Dirac's deltas.

The solution is to superpose a set of $N_{x}$ small Gaussian distributions of $\tilde{\sigma}_{x}$ standard deviation, equally-spaced by an opportune $\Delta x$. In conformity with the treatment of section A.1, it holds

$$
g\left(x ; \mu_{x}, \sigma_{x}\left(z_{s}\right)\right) \simeq \sum_{i=-\frac{N_{x}-1}{2}}^{+\frac{N_{x}-1}{2}} g_{x, i} g\left(x ; \mu_{x}+i \Delta x, \tilde{\sigma}_{x}\right) .
$$

Incorporating (A.15) in (A.14), the final decomposition formula for the transverse phase space density over $\left(x, x^{\prime}\right)$ is obtained:

$$
\begin{aligned}
g\left(x, x^{\prime} ; z\right) \simeq \sum_{i^{\prime}=-\frac{N_{x^{\prime}}-1}{2}} g_{x^{\prime}, i^{\prime}} \delta\left(x^{\prime}-i^{\prime} \Delta x^{\prime}\right) . \\
\\
\\
\quad \sum_{i=-\frac{N_{x}-1}{2}}^{+\frac{N_{x}-1}{2}} g_{x, i} g\left(x ; \mu_{x}=\left(z-z_{s}\right) x^{\prime}+i \Delta x, \tilde{\sigma}_{x}\right) .
\end{aligned}
$$

Therefore, in this subspace the beam is described by $N_{x} \times N_{x^{\prime}}$ identical, zero-divergence beamlets having $\tilde{\sigma}_{x}$ as Gaussian spot size in air. The $\left(i, i^{\prime}\right)$ beamlet is centered at $x=\left(z-z_{s}\right) i^{\prime} \Delta x^{\prime}+i \Delta x$ and moves along $x$ with rate $x^{\prime}=i^{\prime} \Delta x^{\prime}$.

Exploiting (A.16) in the transverse superposition of (27), it results: 


$$
\begin{aligned}
\xi_{\mathrm{B}}^{0}(\vec{r}) \simeq \sum_{i i^{\prime} j j^{\prime}} g_{x, i} g_{x^{\prime}, i^{\prime}} g_{y, j} g_{y^{\prime}, j^{\prime}} . \\
\xi_{\mathrm{L}}^{0}\left(\left|\vec{u}_{0, i^{\prime} j^{\prime}} \times\left(\vec{r}-\vec{r}_{0, i i^{\prime} j^{\prime}}\right)\right|, \vec{u}_{0, i^{\prime} j^{\prime}} \cdot\left(\vec{r}-\vec{r}_{0, i^{\prime} j^{\prime} j^{\prime}}\right)\right),
\end{aligned}
$$

where

$$
\begin{gathered}
\vec{r}_{0, i i^{\prime} j^{\prime}}=\left(\left(z^{*}-z_{s, x}\right) i^{\prime} \Delta x^{\prime}+i \Delta x,\left(z^{*}-z_{s, y}\right) j^{\prime} \Delta y^{\prime}+j \Delta y, 0\right), \\
\vec{u}_{0, i^{\prime} j^{\prime}}=\frac{1}{\sqrt{\left(i^{\prime} \Delta x^{\prime}\right)^{2}+\left(j^{\prime} \Delta y^{\prime}\right)^{2}+1}}\left(i^{\prime} \Delta x^{\prime}, j^{\prime} \Delta y^{\prime}, 1\right) .
\end{gathered}
$$

\section{References}

Andisheh B, Edgren M, Belkić D, Mavroidis P, Brahme A, Lind B K 2013 A comparative analysis of radiobiological models for cell surviving fractions at high doses Technol. Cancer Res. Treat. 12 183-92

Battistoni G, Cerutti F, Fassò A, Ferrari A, Muraro S, Ranft J, Roesler S and Sala P R 2007 The FLUKA code: description and benchmarking AIP Conf. Proc. vol 896 pp 31-49

Böhlen T T, Brons S, Dosanjh M, Ferrari A, Fossati P, Haberer T, Patera V and Mairani A 2012 Investigating the robustness of ion beam therapy treatment plans to uncertainties in biological treatment parameters Phys. Med. Biol. 577983

Bourhaleb F et al 2008 Monte Carlo simulations of ripple filters designed for proton and carbon ion beams in hadrontherapy with active scanning technique J. Phys.: Conf. Ser. 102012002

Britten R A, Nazaryan V, Davis L K, Klein S B, Nichiporov D, Mendonca M S, Wolanski M, Nie X, George J and Keppel C 2013 Variations in the RBE for cell killing along the depth-dose profile of a modulated proton therapy beam Radiat. Res. 179 21-8

Carabe A, Moteabbed M, Depauw N, Schuemann J and Paganetti H 2012 Range uncertainty in proton therapy due to variable biological effectiveness Phys. Med. Biol. 57 1159-72

Chanrion M A, Ammazzalorso F, Wittig A, Engenhart-Cabillic R and Jelen U 2013 Dosimetric consequences of pencil beam width variations in scanned beam particle therapy Phys. Med. Biol. 58 3979-93

Clasie B, Depauw N, Fransen M, Gomà C, Panahandeh H R, Seco J, Flanz J B and Kooy H M 2012 Golden beam data for proton pencil-beam scanning Phys. Med. Biol. 57 1147-58

Dasu A and Toma-Dasu I 2013 Impact of variable RBE on proton fractionation Med. Phys. 40 1-9

Elsässer T and Scholz M 2007 Cluster effects within the local effect model Radiat. Res. 167 319-29

Elsässer T et al 2010 Quantification of the relative biological effectiveness for ion beam radiotherapy: direct experimental comparison of proton and carbon ion beams and a novel approach for treatment planning Int. J. Radiat. Oncol. Biol. Phys. 78 1177-83

Ferrari A, Sala P R, Fassò A and Ranft J 2005 FLUKA: a multi-particle transport code CERN-2005-10, INFN/TC_05/11, SLAC-R-773

Frese M C, Wilkens J J, Huber P E, Jensen A D, Oelfke U and Taheri-Kadkhoda Z 2011 Application of constant versus variable relative biological effectiveness in treatment planning of intensitymodulated proton therapy Int. J. Radiat. Oncol. Biol. Phys. 79 80-8

Friedrich T, Scholz U, Elsässer T, Durante M, Scholz M 2012 Calculation of the biological effects of ion beams based on the microscopic spatial damage distribution pattern Int. J. Radiat. Biol. 88 103-7

Fuchs H, Ströbele J, Schreiner T, Hirtl A and Georg D 2012 A pencil beam algorithm for helium ion beam therapy Med. Phys. 39 6726-37

Gerweck L E and Kozin S V 1999 Relative biological effectiveness of proton beams in clinical therapy Radiotherapy and Oncology 50 135-42

Giordanengo $\mathrm{S}$ et al 2015 The CNAO dose delivery system for modulated scanning ion beam radiotherapy Med. Phys. 42263

Grevillot L, Bertrand D, Dessy F, Freud N and Sarrut D 2011 A Monte Carlo pencil beam scanning model for proton treatment plan simulation using GATE/GEANT4 Phys. Med. Biol. 56 5203-19 
Grevillot L, Bertrand D, Dessy F, Freud N and Sarrut D 2012 GATE as a GEANT4-based Monte Carlo platform for the evaluation of proton pencil beam scanning treatment plans Phys. Med. Biol. $574223-44$

Gueulette J and Wambersie A 2007 Comparison of the methods of specifying carbon ion doses at NIRS and GSI J. Radiat. Res. 48 A97-102

Hall E J and Giaccia A J 2006 Radiobiology for the Radiologist 6th edn (Philadelphia, PA: Lippincott Williams \& Wilkins) p 327

Hawkins R B 2009 The relationship between the sensitivity of cells to high-energy photons and the RBE of particle radiation used in radiotherapy Radiat. Res. 172 761-76

IAEA-TRS461 2008 Relative Biological Effectiveness in Ion Beam Therapy (Technical Report Series) (Vienna: International Atomic Energy Agency)

ICRU Report 161970 Linear Energy Transfer (Bethesda, MD: International Commission on Radiation Units and Measurements)

ICRU Report 301979 Quantitative Concepts and Dosimetry in Radiobiology (Bethesda, MD: International Commission on Radiation Units and Measurements)

ICRU Report 782007 Prescribing, Recording, and Reporting Proton-Beam Therapy (Bethesda, MD: International Commission on Radiation Units and Measurements)

ICRU Report 85a 2011 Fundamental Quantities and Units for Ionizing Radiation (Bethesda, MD: International Commission on Radiation Units and Measurements) (revised)

Kanematsu N, Komori M, Yonai S and Ishizaki A 2009 Dynamic splitting of gaussian pencil beams in heterogeneity-correction algorithms for radiotherapy with heavy charged particles Phys. Med. Biol. 54 2015-27

Kase Y, Kanai T, Matsufuji N, Furusawa Y, Elsässer T and Scholz M 2008 Biophysical calculation of cell survival probabilities using amorphous track structure models for heavy-ion irradiation Phys. Med. Biol. 53 37-59

Kempe J, Gudowska I and Brahme A 2007 Depth dose and LET distributions of ${ }^{1} \mathrm{H},{ }^{4} \mathrm{He},{ }^{7} \mathrm{Li}$ and ${ }^{12} \mathrm{C}$ ions in therapeutic light ion beams Med. Phys. 34 183-92

Kurz C, Mairani A and Parodi K 2012 First experimental-based characterization of oxygen ion beam depth dose distributions at the Heidelberg ion-beam therapy center Phys. Med. Biol. 575017

Krämer M, Jäkel O, Haberer T, Kraft G, Schardt D and Weber U 2000 Treatment planning for heavy-ion radiotherapy: physical beam model and dose optimization Phys. Med. Biol. 45 3299-317

Krämer M and Scholz M 2000 Treatment planning for heavy-ion radiotherapy: calculation and optimization of biologically effective dose Phys. Med. Biol. 45 3319-30

Krämer M and Scholz M 2006 Rapid calculation of biological effects in ion radiotherapy Phys. Med. Biol. 51 1959-70

Krämer M, Wang J F and Weyrather W 2003 Biological dosimetry of complex ion radiation fields Phys. Med. Biol. 48 2063-70

Lin L, Ainsley C G, Mertens T, De Wilde O, Talla P T and McDonough J E 2013 A novel technique for measuring the low-dose envelope of pencil-beam scanning spot profiles Phys. Med. Biol. 58 N171-80

Lomax A 1999 Intensity modulation methods for proton radiotherapy Phys. Med. Biol. 44 185-205

Tessonnier T, Mairani A, Cappucci F, Mirandola A, Vilches Freixas G, Molinelli S, Donetti M and Ciocca M 2014 Development and application of tools for Monte Carlo based simulations in a particle beam radiotherapy facility Appl. Radiat. Isot. 83 155-8

Paganetti H 2009 Dose to water versus dose to medium in proton beam therapy Phys. Med. Biol. 54 4399-421

Pedroni E 2005 Experimental characterization and physical modelling of the dose distribution of scanned proton pencil beams Phys. Med. Biol. 50 541-61

Sawakuchi G O, Zhu X R, Poenisch F, Suzuki K, Ciangaru G, Titt U, Anand A, Mohan R, Gillin M T and Sahoo N 2010 Experimental characterization of the low-dose envelope of spot scanning proton beams Phys. Med. Biol. 55 3467-78

Schaffner B, Pedroni E and Lomax A 1999 Dose calculation models for proton treatment planning using a dynamic beam delivery system: an attempt to include density heterogeneity effects in the analytical dose calculation Phys. Med. Biol. 44 27-41

Shibamoto Y, Otsuka S, Iwata H, Sugie C, Ogino H and Tomita N 2012 Radiobiological evaluation of the radiation dose as used in high-precision radiotherapy: effect of prolonged delivery time and applicability of the linear-quadratic model J. Radiat. Res. 53 1-9 
Soukup M, Fippel M and Alber M 2005 A pencil beam algorithm for intensity modulated proton therapy derived from monte carlo simulations Phys. Med. Biol. 50 5089-104

Steinsträter O, Grun R, Scholz U, Friedrich T, Durante M and Scholz M 2012 Mapping of RBE-weighted doses between HIMAC- and LEM-based treatment planning systems for carbon ion therapy Int. $J$. Radiat. Oncol. Biol. Phys. 84 854-60

Szymanowski H and Oelfke U 2002 Two-dimensional pencil beam scaling: an improved proton dose algorithm for heterogeneous media Phys. Med. Biol. 47 3313-30

Tilly N, Johansson J, Isacsson U, Medin J, Blomquist E, Grusell E and Glimelius B 2005 The influence of RBE variations in a clinical proton treatment plan for a hypopharynx cancer Phys. Med. Biol. 50 $2765-77$

Uzawa A et al 2009 Comparison of biological effectiveness of carbon-ion beams in Japan and Germany Int. J. Radiat. Oncol. Biol. Phys. 73 1545-51

Weber U and Kraft G 1999 Design and construction of a ripple filter for a smoothed depth dose distribution in conformal particle therapy Phys. Med. Biol. 44 2765-75

Zaider M and Rossi H H 1980 The synergistic effects of different radiations Radiat. Res. 83 732-9 